\title{
Rules for designing protein fold switches and their implications for the folding code
}

Yingwei Chen ${ }^{a}$, Yanan $\mathrm{He}^{\mathrm{b}}$, Biao Ruan ${ }^{\mathrm{a}}$, Eun Jung Choi ${ }^{\mathrm{a}}$, Yihong Chen ${ }^{\mathrm{b}}$, Dana Motabar ${ }^{\mathrm{a}, \mathrm{c}}$, Tsega Solomon $^{\mathrm{b}, \mathrm{d}}$, Richard Simmerman ${ }^{\mathrm{a}}$, Thomas Kauffman ${ }^{\mathrm{b}, \mathrm{d}}$, D. Travis Gallagher ${ }^{\mathrm{b}, \mathrm{e}}$, John Orban ${ }^{\mathrm{b}, \mathrm{d}}$, and Philip N. Bryan ${ }^{\mathrm{a}, \mathrm{b}}$

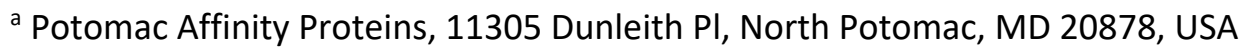

${ }^{b}$ Institute for Bioscience and Biotechnology Research, University of Maryland, 9600 Gudelsky Drive Rockville, MD 20850, USA

${ }^{\mathrm{C}}$ Department of Bioengineering, University of Maryland, College Park, Maryland, 20742, USA

d Department of Chemistry and Biochemistry, University of Maryland, College Park, Maryland, 20742, USA

e National Institute of Standards and Technology and the University of Maryland, 9600 Gudelsky Drive, Rockville Maryland 20850 USA

* To whom correspondence should be addressed: Philip N. Bryan, John Orban Email: pbryan@potomac-affinity-proteins.com jorban@umd.edu

Author Contributions: Yw.C., Y.H., and B.R. contributed equally. Protein design: Yw.C., B.R., E.C., J.O, P.B.; Protein purification and characterization: B.R., Yw.C., E.C., D.M., R.S., D.G., P.B.; Performed NMR structure determination and analysis: Yh.C., Y.H., J.O.; Wrote paper: J.O. (NMR and structural analysis sections), Yw.C., B.R., P.B. (remaining sections).

Competing Interest Statement: Potomac Affinity Proteins sells reagents for protein purification referenced in this study.

Classification: Biological Sciences: Biochemistry; Physical Sciences: Biophysics and Computational Biology

Keywords: protein fold switching, metamorphic, design, NMR structure, evolution 


\begin{abstract}
We have engineered switches between the three most common small folds, $3 \alpha, 4 \beta+\alpha$, and $\alpha / \beta$-plait, referred to here as $A, B$, and $S$, respectively. Mutations were introduced into the natural $S$ protein until sequences were created that have a stable $S$-fold in their longer ( $\sim 90$ amino acid) form and have an alternative fold (either A or B) in their shorter (56 amino acid) form. Five sequence pairs were designed and key structures were determined using NMR spectroscopy. Each protein pair is $100 \%$ identical in the 56 amino acid region of overlap. Several rules for engineering switches emerged. First, designing one sequence with good native state interactions in two folds requires care but is feasible. Once this condition is met, fold populations are determined by the stability of the embedded A- or B-fold relative to the S-fold and the conformational propensities of the ends that are generated in the switch to the embedded fold. If the stabilities of the embedded fold and the longer fold are similar, conformation is highly sensitive to mutation so that even a single amino acid substitution can radically shift the population to the alternative fold. The results provide insight into why dimorphic sequences can be engineered and sometimes exist in nature, while most natural protein sequences populate single folds. Proteins may evolve toward unique folds because dimorphic sequences generate interactions that destabilize and can produce aberrant functions. Thus, two-state behavior may result from nature's negative design rather than being an inherent property of the folding code.
\end{abstract}

\title{
Significance Statement
}

We establish general rules for designing protein fold switches by engineering dimorphic sequences that link the three most common small folds. The fact that switches can be engineered in arbitrary and common protein folds, sheds light on several important questions: 1) What is the generality of fold switching? 2) What types of folds are amenable to switching? 3) What properties are shared by sequences that can fold into two completely different structures? This work has implications for understanding how amino acid sequence encodes structure, how proteins evolve, how mutation is related to disease, and how function is annotated to sequences of unknown structure. 


\section{Introduction}

Fold switching occurs when one amino acid sequence has a propensity for two completely different, but well-ordered, conformations. Many examples of both natural and engineered fold switching demonstrate that proteins can have a stable native fold while simultaneously hiding latent propensities for alternative states with new functions (1-7). This fact has many implications for understanding how amino acid sequence encodes structure, how proteins evolve, how mutation is related to disease, and how function is annotated to sequences of unknown structure. Even as structure prediction has improved, however, detection of latent propensities in a given sequence and prediction of fold switches is usually problematic.

In this paper, we seek to establish some general rules for designing fold switches by engineering switches between three common folds. Our previous studies examined switches between the $3 \alpha\left(G_{A}\right)$ and the $4 \beta+\alpha\left(G_{B}\right)$ domains of Protein $G$ and demonstrated that protein structure can be encoded by a small number of essential residues, and a very limited subset of intra-protein interactions can tip the balance from one fold and function to another $(8,9)$. Here we determine that both $G_{A}$ and $G_{B}$ can switch into a third fold ( $\alpha / \beta$-plait), thus connecting three folds in mutational pathways that avoid unfolded states. The premise of this paper is that if switches can be engineered in arbitrary and common protein folds, it will shed light on several important questions: 1) What is the generality of fold switching? 2) What types of folds are amenable to switching? 3) What properties are shared by sequences that can fold into two completely different structures?

The proteins used in this study have no significant homology, represent the three most common fold types (10), and are models for studying protein folding and stability (Fig. S1). All 
are small and amenable to NMR studies. They did not pass any initial test of likely switching. By studying small proteins that are widely used in experimental and computational folding studies, experimental results connect a large body of knowledge e.g. (11-24). Streptococcal Protein G contains two types of domains that bind to serum proteins in blood: the $G_{A}$ domain binds to human serum albumin $(\mathrm{HSA})(25,26)$ and the $\mathrm{G}_{B}$ domain binds to the constant $(\mathrm{Fc})$ region of $\operatorname{IgG}$ $(27,28)$. The ribosomal protein $\mathrm{S} 6$ from Thermus thermophilus is a well-studied member of the $\alpha / \beta$-plait family (29-33). For simplicity, the $S 6$ fold is referred to as an S-fold. When a switch to either the $G_{A}$ or $G_{B}$ fold is discussed, both are referred to as a $G$-fold. The specific $G_{A}$ fold is referred to as an A-fold and the specific $G_{B}$ fold is referred to as a B-fold.

The basic challenge in designing fold switches is, given two arbitrary folds, how do you identify one sequence that contains the essential folding information for both folds? Theoretically, a simple approach is an exhaustive computational search to find one sequence that has mutually compatible native interactions in two conformations. A more practical design process requires a method for aligning the sequences for the two folds such that essential folding information for both folds can be introduced by mutation in a way that is mutually compatible. For example, automated alignment can thread the shorter sequence through the larger structure and calculate the energy of hypothetical structures in every register (34). This approach is not dependable for designing switches, however, because some alignments with high energies can be radically improved with a few strategic mutations. To maximize possibilities of a parsimonious switch, we aligned the sequences in all registers and evaluated what would have to change to accommodate both folds. The process was as follows:

i. Thread a G-sequence through the S-fold in each possible alignment. 
ii. Identify alignments that minimize the number of catastrophic interactions.

iii. Determine tolerable mutations in the $\mathrm{G}$-sequence that might resolve the catastrophic clashes in the S-fold. Redesign clusters of amino acids to resolve clashes. Use the Rosetta-Relax protocol to adjust the peptide backbone and evaluate the energy of the design (35).

iv. Optimize protein stability in the S-fold by computationally mutating amino acids at nonoverlapping positions. Repeat minimization and evaluation with Rosetta-Relax. To minimize uncertainties involved in computational design, conserve original amino acids whenever possible.

Previously we created sequences that populate both A- and B-folds by threading the Asequence through the B-fold, finding a promising alignment, and then using phage-display selection to reconcile one sequence to both folds $(8,36,37)$. Here the approach is conceptually similar, except that we use Rosetta as a computational design tool to identify compatible mutations rather than phage display. There is no reason to assume that this method is optimal. We are merely applying a practicable scheme for engineering dimorphic sequences and then evaluating the outcome using structure determination by NMR and thermal denaturation.

Five dimorphic sequence pairs (10 proteins) were designed and purified (Fig. 1). In each of these designs, the protein pairs are $100 \%$ identical in a 56 amino acid region of overlap. Analysis of thermal denaturation showed eight of the 10 to be stable, well-populated structures. Seven structures were determined using NMR spectroscopy and compared to the designs. Two of the switches (one S- to A-switch and one S- to B-switch) achieved the goal of populating the Sfold in the longer form and the A- or B-fold in the shorter form. The other cases were equally informative, however. Here, we describe the folding energetics and structures of these 10 
dimorphic proteins and present a set of basic principles for designing fold switches that emerged from this analysis.

\section{Results}

Design of $S_{a 1}$ and $A_{1}$. Designing a dimorphic sequence is an iterative process. After examining the 40 possible alignments of the 56 amino acid A-sequence in the 95 amino acid Sfold, we chose amino acids $11-66$ as the preferred region of overlap (Fig. S2). This alignment generated nine positions of identity between the starting sequences. In terms of topological alignment, $\alpha 1(\mathrm{~S})$ mostly coincides with $\alpha 1(\mathrm{~A})$. $\beta 2(\mathrm{~S})$ becomes $\alpha 2(\mathrm{~A})$, and the long $\beta 2-\beta 3$ turn and first half of $\beta 3(\mathrm{~S})$ becomes $\alpha 3(\mathrm{~A}) . \quad \beta 1$ and $\alpha 2-\beta 4$ of the S-fold are outside of the overlap region. Mutations to resolve catastrophic interactions in this alignment were designed in clusters of 4-6 amino acids using Pymol (38) and relaxed structures were generated using Rosetta-Relax (35). Ultimately, we made 25 substitutions of an S-residue with an A-residue, substituted with a third choice in 5 cases, and retained the S-residue at 26 positions. We then examined the non-overlapping region of sequence and made 14 additional mutations to generate the $S_{a 1}$ sequence. The 56 amino acid version of the protein has 22 total changes: 17 substitutions of an A- with the S-residue and 5 changes to a third choice (Fig. S2). The final computational models for $S_{a 1}$ and $A_{1}$ were generated by Rosetta using the Relax application. The Relax protocol searches the local conformational space around the native, experimentallydetermined structure and is used only to evaluate whether the designed mutations have favorable native interactions within that limited conformational space. The designed models of $S_{a 1}$ and $A_{1}$ show relatively small increases in energy compared to the relaxed native structures (Supplemental PDB files of the Rosetta models). 
Structure of $A_{1}$. Overall, the $3 \alpha$-helical bundle topology of $A_{1}$ is very similar to the $G_{A}$ parent structure from which it was derived (39). The sequence specific chemical shift assignments for $A_{1}$ (Fig. 2A) were utilized to calculate a 3D structure with CS-Rosetta (Fig. 2B, Table S1). Our previous studies indicated close correspondence of CS-Rosetta and de novo structures for A- and B-folds (40). The N-terminal residues 1-4 and the C-terminal residues 53-56 are disordered in the structure, consistent with $\left\{{ }^{1} \mathrm{H}\right\}{ }^{15} \mathrm{~N}$ steady state heteronuclear NOE data (Fig. 2D).

Structure of $S_{a 1}$. Likewise, $S_{a 1}$ has the same overall $\beta \alpha \beta \beta \alpha \beta$-topology as the parent $\mathrm{S} 6$ structure (Fig. 2C, Table S2). The backbone chemical shifts (Fig. 2A) were used in combination with main chain inter-proton NOEs (Fig. S3) to determine a three-dimensional structure using CS-Rosetta. The conformational ensemble shows well-defined elements of secondary structure at residues 2-10 ( $\beta 1), 16-32(\alpha 1), 40-44(\beta 2), 59-67(\beta 3), 73-81(\alpha 2)$ and 86-92 ( $\beta 4)$. The principal difference from the native structure is that the $\beta 2$-strand is seven amino acids shorter in $\mathrm{S}_{\mathrm{a} 1}$ than in S6. Heteronuclear NOE data show overall consistency with the structure, indicating that the loop between the $\beta 2$ - and $\beta 3$-strands from residues $45-58$ is more flexible than other internal regions of the polypeptide chain (Fig. 2D).

Although the 56 amino acid sequence of $A_{1}$ is $100 \%$ identical to residues $11-66$ of $S_{a 1}$ a significant fraction of the residues undergo large amplitude changes in their backbone $\phi / \psi$ torsion angles between these two structures (Fig. 3A). Amino acids 1-4 form the disordered Nterminal tail in $A_{1}$ and are part of the loop between the $\beta 1$-strand and $\alpha 1$-helix in $S_{a 1}$ (residues 11-14). Amino acids 5-23 form the $\alpha 1$-helix in $A_{1}$ and the equivalent sequence in $S_{a 1}$ (residues 15-33) forms a similar length $\alpha 1$-helix. Amino acids $24-26$ form the loop between the $\alpha 1$ - and 
$\alpha 2$-helices in $A_{1}$ and form the first part of the loop between the $\alpha 1$-helix and $\beta 2$-strand in $S_{a 1}$ (residues 34-36). Amino acids $27-35$ in the $\alpha 2$-helix of $A_{1}$ correspond with the extended part of the $\alpha 1-\beta 2$ loop and the $\beta 2$ strand in $S_{a 1}$ (residues $37-45$ ). Amino acids $36-38$ form the loop between the $\alpha 2$ - and $\alpha 3$-helices in $A_{1}$ and are part of the loop between the $\beta 2$ - and $\beta 3$-strands in $S_{a 1}$ (residues 46-48). Amino acids 39-56 in the $\alpha 3$-helix and C-terminal tail of $A_{1}$ form a portion of the $\beta 2-\beta 3$ loop and the $\beta 3$-strand in $S_{a 1}$ (residues 49-66).

The CS-Rosetta structures calculated here employ main chain chemical shift and NOE restraints but do not have experimental restraints for side chains. Nevertheless, the overall positions of the core side chains are very likely to be correct to a close approximation due to the packing requirements dictated by the respective folds. It is therefore instructive to compare the location of corresponding modeled side chains for core residues in the $3 \alpha$ versus $\alpha / \beta$-plait folds of these NMR-derived structures. Residues contributing to the core in $A_{1}$ consist of $L 9, A 12, K 13$, A16, I17, L20, and Y23 from the $\alpha 1$-helix; I25 from the loop between the $\alpha 1$ - and $\alpha 2$-helices; I30 and I33 from the $\alpha 2$-helix; and V39, V42, K46, 149, and L50 from the $\alpha 3$-helix (Fig. 3B). The core of $\mathrm{S}_{\mathrm{a} 1}$ is somewhat larger with 21 residues versus 15 residues for $A_{1}$. Core amino acids in the $\alpha 1$ helix of $A_{1}$ correspond with residues that also contribute to the core of $S_{a 1}$. Only two of these amino acids, A16/A26 and L20/L30, are completely buried in both folds. In contrast with the $3 \alpha$ fold of $A_{1}$, the $\alpha 1$-helix in the $\alpha / \beta$-plait fold of $S_{a 1}$ contacts an almost entirely different set of residues. For example, amino acids $L 51, Y 53$, and 155 in the C-terminal tail of $A_{1}$ do not have extensive contacts with $\alpha 1$ but the corresponding residues in $S_{a 1}$ (L61, Y63, and I65) form close core interactions with $\alpha 1$ as part of the $\beta 3$-strand. Most of the other core residues contacting the $\alpha 1$-helix of $S_{a 1}$ are outside the 56 amino acid region coding for the $A_{1}$ fold. These include $F 4$, V6, I8, and L10 from the $\beta 1$-strand; A67 from the $\beta 3$-strand; V72, L75, and L79 from the $\alpha 2$ - 
helix; and V85 from the loop between the $\alpha 2$-helix and the $\beta 4$-strand. Two additional residues, V88 and V90 ( $\beta 4)$ also contribute significantly to the core but do not contact $\alpha 1$. Thus, beyond the original topological alignment of the $\alpha 1$-helices, the cores of the $3 \alpha$ and $\alpha / \beta$-plait folds are largely non-overlapping. In total, approximately half $(11 / 21)$ of the residues participating in the $\mathrm{S}_{\mathrm{a} 1}$ core are not present in the $\mathrm{A}_{1}$ sequence. This includes residues 1-10 at the $\mathrm{N}$-terminus, which contribute 4 amino acids to the $S_{a 1}$ core $(F 4, V 6,18, L 10)$, and residues 67-95 which provide 7 core amino acids (A67, V72, L75, L79, V85, V88, and V90).

$C D$ analysis of unfolding for $A_{1} / S_{a 1}$. Far-UV CD spectra were measured for $S_{a 1}$ and $A_{1}$ and thermal unfolding profiles were determined by measuring ellipticity at $222 \mathrm{~nm}$ vs. temperature (Fig. S4). The fraction native was determined by subtracting an unfolded baseline from the experimental CD signal and then dividing by the total CD difference between $100 \%$ folded and $0 \%$ folded at that temperature. Reversibility of unfolding was confirmed by comparing the $\mathrm{CD}$ spectra at $293^{\circ} \mathrm{K}$ before melting and after heating to $373^{\circ} \mathrm{K}$ and cooling to $293^{\circ} \mathrm{K}$. The temperature unfolding profiles were converted to an apparent $\Delta \mathrm{G}_{\text {folding }}$ and fit to a theoretical curve calculated using the Gibbs-Helmholtz equation: $\Delta \mathrm{G}_{\text {folding }}=\Delta \mathrm{H}_{\mathrm{O}}-\mathrm{T} \Delta \mathrm{S}_{\mathrm{O}}+\Delta \mathrm{Cp}(\mathrm{T}-$ $\mathrm{T}_{\mathrm{O}}-\mathrm{T} \operatorname{lnT} / \mathrm{T}_{\mathrm{O}}$ ), where $\mathrm{T}_{\mathrm{O}}=298^{\circ} \mathrm{K}$ (Fig. 4A, B) (41). $\mathrm{S}_{\mathrm{a} 1}$ has a $\mathrm{T}_{\mathrm{M}}$ of $\sim 373^{\circ} \mathrm{K}$ and an estimated $\Delta \mathrm{G}_{\text {folding }}$ of $-7.5 \mathrm{kcal} / \mathrm{mol}$ at $298^{\circ} \mathrm{K}$ (Fig. $4 \mathrm{~B}, \mathrm{C}$ ). The $\Delta \mathrm{G}_{\text {folding }}$ of the parent $\mathrm{S} 6$ is $-8.5 \mathrm{kcal} / \mathrm{mol}$ (32). The Rosetta energy of the $\mathrm{S}_{\mathrm{a} 1}$ design is similar to the native sequence (Fig. 4D). $\mathrm{A}_{1}$ has a $\Delta \mathrm{G}_{\text {folding }}=$ $-4.0 \mathrm{kcal} / \mathrm{mol}$ at $298^{\circ} \mathrm{K}$. The $\Delta \mathrm{G}_{\text {folding }}$ of the parent is $-5.6 \mathrm{kcal} / \mathrm{mol}(42,43)$. The Rosetta energy of the $A_{1}$ design is slightly more favorable than the native sequence (Fig. 4D).

Design 1 of $S_{b 1}$ and $B_{1}$. After examining the 40 possible alignments of the 56 residue $B$ sequence in the 95 residue S-fold, we chose amino acids 4-59 as the preferred region of overlap 
(Fig. S5). This alignment generated five positions of identity between the starting sequences. In terms of topological alignment, $\beta 1(\mathrm{~S})$ mostly coincides with $\beta 1(\mathrm{~B})$. The first half of $\alpha 1(\mathrm{~S})$ becomes $\beta 2(B)$ and the second half of $\alpha 1(S)$ becomes the first half of $\alpha 1(B)$. The $\beta 2$ strand of $S$ becomes the second half of $\alpha 1(B)$, a turn, and the first part of $\beta 3(B)$. The long $\beta 2-\beta 3(S)$ turn and the first part of $\beta 3(S)$ become the second part of $\beta 3$ and $\beta 4$ of $B$. The second half of $\beta 3$ and $\alpha 2-$ $\beta 4$ of the S-fold are outside of the overlap region. At the 51 positions of non-identity from 4 to 59, we made 40 substitutions of an S-residue with an A-residue, substituted with a third choice in 4 cases, and retained the S-amino acid at 7 positions. We then made 14 additional mutations in the non-overlapping region to generate the $S_{b 1}$ sequence. The 56 amino acid version of the protein has 11 total changes: 7 substitutions of an A-residue with the S-residue and 4 changes to a third choice (Fig. S5). The energies of the computational models for $\mathrm{S}_{\mathrm{b} 1}$ and $\mathrm{B}_{1}$ show relatively small increases in energy compared to the relaxed native structures (Fig. 4D).

Structure of $B_{1}$. The $\beta \beta \alpha \beta \beta$ topology of $B_{1}$ is very similar to that of the parent $B$-fold, with a backbone RMSD of $\sim 0.6 \AA$. The NMR structure consists of four $\beta$-strands defined by residues 2-9 ( $\beta 1)$, residues 13-20 ( $\beta 2)$, residues $42-46(\beta 3)$, and residues $50-55(\beta 4)$ and one $\alpha-$ helix from residues 23-37 (Fig. 5A, Fig. S6B, Table S1).

Structure of $S_{b 1}$. The topology of $S_{b 1}$ is not the same as the parent $S 6$ structure. Instead, the $2 \mathrm{D}^{1} \mathrm{H}-{ }^{15} \mathrm{~N}$ HSQC spectrum of $\mathrm{S}_{\mathrm{b} 1}$ has a pattern similar to that of $\mathrm{B}_{1}$ (Fig. 5A). NMR assignment of the main chain resonances showed the presence of four $\beta$-strands and two $\alpha$-helices, but the order of the secondary structure elements was $\beta \beta \alpha \beta \beta \alpha$ rather than the $\beta \alpha \beta \beta \alpha \beta$ arrangement expected for an S-type fold. Initial NMR structures of $S_{b 1}$ indicated a B-fold, which was supported by backbone NOE connectivities (Fig. S3), with a mostly disordered C-terminal tail. CS-Rosetta 
modeled residues 73-83 near the C-terminus as an $\alpha 2$-helix. Of these, amide signals due to residues 73-80 were not detectable in NMR spectra while residues $81-83$ were helical based on assigned chemical shifts. Comparison of $\mathrm{S}_{\mathrm{b} 1}$ amide chemical shifts with those of $\mathrm{B}_{1}$ indicated that most of the perturbations due to the C-terminal 35 amino acid tail were localized in $\alpha 1, \beta 3$, and neighboring regions (Fig. 5B,E). This suggested that the putative $\alpha 2$-helix interacts with the Bfold in these contiguous regions. Mutations $\mathrm{R} 72 \mathrm{C}$ and $\mathrm{R} 83 \mathrm{C}$ were made at the $\mathrm{N}$ - and $\mathrm{C}$-terminal ends of the $\alpha 2$ region in separate samples of $S_{b 1}$ and these proteins were derivatized with the stable nitroxide spin label MTSL. Paramagnetic relaxation enhancement (PRE) measurements (Fig. 5C) showed significant decreases in amide peak intensity over the $\alpha 1$ and $\beta 3$ regions for the B-core of $S_{b 1}$, consistent with the chemical shift perturbation data. Furthermore, the PRE intensity profiles were similar regardless of which end of the $\alpha 2$ region the spin label resided. This suggests that docking of the $\alpha 2$ region against the $B$-folded core of $S_{b 1}$ is in exchange between multiple states, providing a plausible explanation for why most of the $\alpha 2$ amide resonances are not detectable. Structures for $S_{b 1}$ were re-calculated using additional weak (<20Å) PRE restraints, showing an ensemble with a well-defined B-core that has a putative $\alpha 2$ helix packed against it loosely (Fig. 5D, E). Steady-state $\left\{{ }^{1} \mathrm{H}\right\}-{ }^{15} \mathrm{~N}$ heteronuclear NOE data for $\mathrm{S}_{\mathrm{b} 1}$ were consistent with the structure (Fig. 5F). In particular, the C-terminal tail becomes more ordered around the $\alpha 2$ region, although these heteronuclear NOE values (0.4-0.7) are still below those of well-ordered regions $(>0.8)$. Thus, the structure of $S_{b 1}$ may be viewed as a transitory state between the S- and B-folds. With two $\alpha$-helices packed against a 4-stranded $\beta$-sheet, $\mathrm{S}_{\mathrm{b} 1}$ has the same overall two-layer $\alpha / \beta$-sandwich architecture as the S-fold but differs in the topological arrangement of secondary structures. 
$C D$ analysis of unfolding for $B_{1} / S_{b 1} . B_{1}$ has a $\Delta G_{\text {folding }}=-4.0 \mathrm{kcal} / \mathrm{mol}$ at $298^{\circ} \mathrm{K}$ compared to $-6.6 \mathrm{kcal} / \mathrm{mol}$ for the parent (44). The Rosetta energy of the $\mathrm{B}_{1}$ design is a little less favorable than for the native sequence and generally consistent with its $\Delta \mathrm{G}_{\text {folding }}$ (Fig. 4C, D). In contrast, $\mathrm{S}_{\mathrm{b} 1}$ has a minimum $\Delta \mathrm{G}_{\text {folding }}=-1.1 \mathrm{kcal} / \mathrm{mol}$ at $\sim 298^{\circ} \mathrm{K}$ (Fig. $4 \mathrm{~B}$ ). As described above, the predominant folded form at $298^{\circ} \mathrm{K}$ is the B-conformation. The energy of its Rosetta design in the S-conformation is similar to that of $S_{a 1}$, however. Thus, while $B_{1}$ and $S_{b 1}$ have identical sequences in their respective 56 amino acid B-folds, the 35 residue $\mathrm{C}$-terminal tail in $\mathrm{S}_{\mathrm{b} 1}$ destabilizes the $\mathrm{B}$ fold, presumably by populating competing alternative states. Elucidating the reason for the large inconsistency between the $S_{b 1}$ design energy and the observed fold is critical for switch design and was further investigated below.

Design 2 of $S_{b_{2}}$ and $B_{2}$. We introduced 13 mutations into the $S_{b 1}$ sequence to generate a second dimorphic version in this alignment (Fig. S5). The Rosetta energy of the $\mathrm{S}_{\mathrm{b} 2}$ design model is almost identical to the $S_{b 1}$ design model. The 56 amino acid version of $S_{b 2}$ (denoted $B_{2}$ ) has a significantly higher Rosetta energy than $B_{1}$ (Fig. 4D), however.

Structure of $S_{b 2}$. The 3D structure of $S_{b 2}$ contains four $\beta$-strands and two $\alpha$-helices and has the general features of the parent S-fold (Fig. S6, Table S2). The ordered regions in the

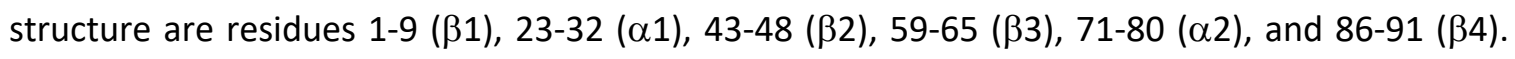
While the parent $S$ (PDB 1RIS) and $S_{a 1}$ structures are very similar, the $S_{b 2}$ structure differs from both in a number of ways despite having the same overall topology. The $\alpha 1$-helix in $S_{b 2}$ is shorter, comprising 10 amino acids compared with 17 amino acids in $\mathrm{S}_{\mathrm{a} 1}$. Also, the $\beta 2$-strand forms 4 amino acids later in the $S_{b 2}$ polypeptide chain than in $S_{a 1}$. The first residue in the $\beta 2$ strand of $S_{b 2}, G 43$, interacts with $E 65$ in the $\beta 3$-strand. This represents a two-residue shift in the 
register of hydrogen bonding between $\beta 2$ and $\beta 3$ in $S_{b 2}$ compared with $S_{a 1}$ (Fig. S3B). As a result of these differences, the loops connecting $\beta 1$ to $\alpha 1$ and $\alpha 1$ to $\beta 2$ are longer in $S_{b 2}(13$ and 10 residues, respectively) than in $S_{a 1}$ ( 5 and 7 residues, respectively). The remainder of the $S_{b 2}$ structure encompassing $\beta 1, \beta 3, \alpha 2$, and $\beta 4$ is very similar to $S_{a 1}$. Heteronuclear NOE dynamics data for $S_{b 2}$ were consistent with the NMR structure (Fig. S6D). In particular, the relatively long $\beta 1-\alpha 1, \alpha 1-\beta 2$, and $\beta 2-\beta 3$ loops were found to be the most flexible on the ns-ps timescale. We were not able to characterize the $B_{2}$ structure because it was largely unfolded, consistent with its increased Rosetta energy. Instead, the structure of $B_{1}$ was used for comparisons with $S_{b 2}$ because the corresponding B-regions also have very high sequence identity (80\%). Detailed comparisons between $\mathrm{B}_{1}$ and $\mathrm{S}_{\mathrm{b} 2}$ are presented in Supplemental Material (Fig. S7, and Supplementary Information).

CD analysis of unfolding for $B_{2} / S_{b 2}$. $S_{b 2}$ has a minimum $\Delta G_{\text {folding }}=-4.0 \mathrm{kcal} / \mathrm{mol}$ at $\sim 298^{\circ} \mathrm{K}$ (Fig. 4B). As described above, the predominant folded form at $298^{\circ} \mathrm{K}$ is the S-conformation. Its Rosetta design energy is actually slightly less favorable than the design energy for $S_{b 1}$, however. From $C D, B_{2}$ appears to be $\geq 95 \%$ unfolded conformation $\left(\Delta \mathrm{G}_{\text {folding }} \geq 2 \mathrm{kcal} / \mathrm{mol}\right.$ ) throughout the temperature range from $278-373^{\circ} \mathrm{K}$, consistent with its unfavorable Rosetta energy (Fig. 4D). Thus the fold switch between $S_{b 1}$ (B-fold) and $S_{b 2}$ (S-fold) appears to result from decreased stability of the embedded B-fold rather than improved interactions in the folded Sconformation.

Design 3 of $S_{b 3}$ and $B_{3}$. Analysis of the NMR structures of $S_{b 1}$ and $S_{b 2}$ provided clues about how to improve the design of dimorphic B-fold/S-fold proteins. In the computational design of $\mathrm{S}_{\mathrm{b} 1}$, the DDATK turn should become part of the long connection between $\beta 2$ and $\beta 3$ of the S-fold. The sequence actually remains in the B-conformation, however. This occurs in spite of 
acceptable native interactions in the S-conformation, as assessed by Rosetta. We did not anticipate that turn propensities would be harder to override than secondary structure propensities but clearly turn sequences (even without proline or glycine) can contain critical topological information (45-48). The $\mathrm{S}_{\mathrm{b} 2}$ sequence had two substitutions in the DDATK sequence which decrease its strong propensity for the short turn. Based on this insight, we redesigned the S-fold to increase its compatibility with the B-fold. The S-fold is classified as a superfold with many natural variations in the length and position of turns (49). In particular, some natural Sfolds (protease inhibitors) have a short turn between $\beta 2$ and $\beta 3$ that matches the B-fold turn between $\beta 3$ and $\beta 4$ (50-52). These protease inhibitors have a longer loop between $\beta 1$ and $\alpha 1$. We made an S-fold of this type by inserting three residues (GTD) between $\beta 1$ and $\alpha 1$ and deleting 12 residues (RQLSEPIAKDPQ) from the long loop between $\beta 2$ and $\beta 3$ (Fig. S8, S9). This creates a topological match between $\alpha 1 \beta 3 \beta 4$ in $B$ and $\alpha 1 \beta 2 \beta 3$ in S.

In this design, amino acids 1-56 are the region of overlap (Fig. S8). In terms of topological alignment, the $\beta 1-$ strand is the same in both folds but changes orientation, the long turn between $\beta 1$ and $\alpha 1$ in $S_{b 3}$ becomes $\beta 2$ of the $B$-fold, and the $\alpha 1-\beta 2-\beta 3$ of $S_{b 3}$ maintains the same topology in the $B_{3}$ design. The $\alpha 2$-helix and the $\beta 4$-strand of $S_{b 3}$ are outside the overlap region. At the 47 positions of non-identity, we made 33 substitutions of an S-residue with a Bresidue, substituted with a third choice in 12 cases, and retained the S-amino acid at one position. We then made 18 additional mutations in the non-overlapping region to generate the $\mathrm{S}_{\mathrm{b} 3}$ sequence. The 56 amino acid version of the protein has 12 total changes: 1 substitution of a B-residue with the S-residue and 11 changes to a third choice. The energy of the computational model for $S_{b 3}$ is slightly more favorable than the relaxed native structure. The designed model 
for $B_{3}$ has a less favorable energy than the native $B$-sequence but is more favorable than the relaxed $\mathrm{B}_{1}$ design (Fig. 4D).

Structural analysis of $\mathrm{B}_{3}$. The $2 \mathrm{D}{ }^{1} \mathrm{H}-{ }^{15} \mathrm{~N}$ HSQC spectrum of $\mathrm{B}_{3}$ at $278^{\circ} \mathrm{K}$ and low concentrations $(<20 \mu \mathrm{M})$ was consistent with a predominant, monomeric B-fold (Fig. S10) but showed significant exchange broadening at $298^{\circ} \mathrm{K}$, indicative of low stability (see below). Presumably the low stability is due to less favorable packing of $\mathrm{Y} 5$ in the core of the B-fold compared with a smaller aliphatic leucine. However, additional, putatively oligomeric, species were also present for which relative peak intensities increased with increasing protein concentration. Due to its relatively low stability and sample heterogeneity, $B_{3}$ was not analyzed further structurally.

Structural analysis of $S_{b 3}$. In contrast, when the 56-residue $B_{3}$ sequence was embedded in the longer 87-residue polypeptide chain to give $S_{b 3}$, it provided a homogeneous sample for which the HSQC spectrum was readily assigned (Fig. 6A). NMR-based structure determination indicated that $\mathrm{S}_{\mathrm{b} 3}$ has a $\beta \alpha \beta \beta \alpha \beta$ secondary structure and an S-fold topology (Fig. 6C). Ordered regions correspond with residues 4-10 ( $\beta 1), 24-37(\alpha 1), 42-46(\beta 2), 51-56(\beta 3), 62-70(\alpha 2)$, and 79-85 ( $\beta 4)$. Comparison of $S_{b 3}$ with the parent S-fold indicates that the $\beta 1 / \alpha 2 \beta 4$ portion of the fold is similar in both. In contrast, the $\beta 1-\alpha 1$ loop is longer in $S_{b 3}(13$ residues) than in the parent S-fold (5 residues), while $\alpha 1, \beta 2$, the $\beta 2-\beta 3$ loop, and $\beta 3$ are all shorter than in the parent (Fig. 6C). Consistent with the $S_{b 3}$ structure, the 13 amino acid $\beta 1-\alpha 1$ loop is highly flexible (Fig. 6D).

CD analysis of unfolding for $B_{3} / S_{b 3}$. $S_{b 3}$ has a minimum $\Delta G_{\text {folding }}=-3.5 \mathrm{kcal} / \mathrm{mol}$ at $\sim 298^{\circ} \mathrm{K}$ (Fig. 4B,C). As described above, the predominant form is an S-fold. The Rosetta energy of its design is slightly more favorable than the energy of the $S_{a 1}$ design even though the stability of its 
S-fold is less by $\sim 4 \mathrm{kcal} / \mathrm{mol}$ (Fig. 4C,D). $B_{3}$ has a $\Delta G_{\text {folding }}$ of $-1.2 \mathrm{kcal} / \mathrm{mol}$ at $298^{\circ} \mathrm{K}$ (Fig. 4A,C). The $\Delta \mathrm{G}_{\text {folding }}$ of $\mathrm{B}_{3}$ is less than would be expected from its Rosetta energy. From the NMR analysis, it appears that the B-fold is in equilibrium with putatively dimeric states. This creates a situation in which the B-fold is both temperature dependent and concentration dependent. The predominant form at $278^{\circ} \mathrm{K}$ and $\leq 18 \mu \mathrm{M}$ is the B-fold, however. The low stability and concentration-dependent behavior of $B_{3}$ may indicate that some propensity for the $S$ conformation could persist in the 56-residue protein.

Design of $\mathrm{S}_{b 4}$ and $\mathrm{B}_{4}$. We used the NMR structure of $\mathrm{S}_{\mathrm{b} 3}$ to design a point mutation (Y5L) that would stabilize the embedded B-fold and simultaneously destabilize the S-fold. This was expected to shift the population to the B-fold. The $\mathrm{Y} 5 \mathrm{~L}$ mutation was also introduced into $\mathrm{B}_{3}$ to determine its effect on the stability of the B-fold in the 56 amino acid protein. These new dimorphic proteins are denoted $\mathrm{S}_{\mathrm{b} 4}$ and $\mathrm{B}_{4}$.

Structural analysis of $B_{4}$. Assignment and structure determination of $B_{4}$ showed its topology to be identical to the parent B-topology (Fig. 6A, B). At concentrations above $100 \mu \mathrm{M}$, $\mathrm{B}_{4}$ displayed a tendency for weak self-association similar to that seen for $\mathrm{B}_{3}$.

Structural analysis of $S_{b 4}$. Incorporation of the single amino acid change $Y 5 L$ into $S_{b 3}$ to give $S_{b 4}$ resulted in approximately twice the number of amide cross-peaks in the HSQC spectrum relative to the $S_{b 3}$ sample. Comparison of the spectrum of $S_{b 4}$ with spectra of $S$ - and B-folds for the closely related sequences of $B_{4}$ and $S_{b 3}$ indicated that $S_{b 4}$ populates both $S$ - and B-states simultaneously in an approximately 1:1 ratio at $298^{\circ} \mathrm{K}$ (Fig. S11). Due to this heterogeneity, the structure of $S_{b 4}$ was not analyzed further here.

Comparison of $\mathrm{S}_{\mathrm{b} 3}$ and $\mathrm{B}_{4}$. The aligned amino acid sequences of $\mathrm{S}_{\mathrm{b} 3}$ and $\mathrm{B}_{4}$ show that 
their B-regions have $98 \%$ sequence identity (Fig. S8), the only difference being an L5Y mutation in $S_{b 3}$. The global folds of $S_{b 3}$ and $B_{4}$ have large-scale differences, however (Fig. 7A). The $\beta 1$ strands, while similar in length, are in opposite directions in $S_{b 3}$ and $B_{4}$. The $\beta 1$-strand forms a parallel stranded interaction with $\beta 4$ in $\mathrm{B}_{4}$, but an antiparallel interaction with the corresponding $\beta 3$-strand in $\mathrm{S}_{\mathrm{b} 3}$. Whereas residues 9-20 form the 6-residue $\beta 1-\beta 2$ turn and the 6-residue $\beta 2$ strand of $B_{4}$, these amino acids constitute the end of $\beta 1$ and 10 residues of the large disordered $\beta 1-\alpha 1$ loop in $S_{b 3}$. The remainder of the B-region is topologically similar, with the $\alpha 1 / \beta 3 / \beta 4$ structure in $\mathrm{B}_{4}$ matching the $\alpha 1 / \beta 2 / \beta 3$ structure in $\mathrm{S}_{\mathrm{b} 3}$. Overall, however, the order of $\mathrm{H}$-bonding in the 4-stranded $\beta$-sheets is quite different, with $\beta 2 \beta 3 \beta 1 \beta 4$ in $S_{b 3}$ and $\beta 3 \beta 4 \beta 1 \beta 2$ in $B_{4}$.

The main core residues of $B_{4}$ consist of $Y 3, L 5, L 7$, and $L 9$ from $\beta 1, A 26, F 30$, and $A 34$ from $\alpha 1$, and $F 52$ and $V 54$ from $\beta 4$ (Fig. 7B). In $S_{b 3}$, the topologically equivalent regions of the core are $\mathrm{A} 26, \mathrm{~F} 30$, and $\mathrm{A} 34$ from $\alpha 1$, and $\mathrm{F} 52$ and $\mathrm{V} 54$ from $\beta 3$. Residues $\mathrm{Y} 5, \mathrm{~L} 7$, and $\mathrm{L} 9$ from the $\beta 1$ strand of $S_{b 3}$ also form part of the core, but with different packing from $B_{4}$ due to the reverse orientation of $\beta 1$. Residues $A 12$ and $A 20$, which contribute to the periphery of the core in $B_{4}$, are solvent accessible in the $\beta 1-\alpha 1$ loop of $S_{b 3}$. Most of the remaining core residues of $S_{b 3}$ come from outside of the B-region and include amino acids from $\beta 3$ (A56), $\alpha 2$ (V64, L67, A68, L71), and $\beta 4$ (V80 and 182). Overall, the degree of overlap between the cores of $B_{4}$ and $S_{b 3}$ is higher than for $A_{1} / S_{a 1}$ and $B_{1} / S_{b 2}$ (compare Figs. 3, 6, and $S 7$ ), indicating that while mutual exclusivity of cores may be advantageous for fold switching it is not an absolute requirement.

$C D$ analysis of unfolding for $B_{4} / S_{b 4}$. Thermal denaturation by $C D$ shows that $B_{4}$ has $a$ $\Delta \mathrm{G}_{\text {folding }}=-4.1 \mathrm{kcal} / \mathrm{mol}$ at $298^{\circ} \mathrm{K}$ (Fig. $4 \mathrm{~A}, \mathrm{C}$ ). The stability of $\mathrm{S}_{\mathrm{b} 4}$ can be derived from the NMR analysis because $\mathrm{S}$ - and $\mathrm{B}$-folds are observed in equal mixture. Thus, the $\Delta \mathrm{G}_{\text {folding }}$ for both the $\mathrm{S}$ - 
and $\mathrm{B}$-folds of $\mathrm{S}_{\mathrm{b} 4}$ is $\sim 0 \mathrm{kcal} / \mathrm{mol}$. Appending the 31 residue $\mathrm{C}$-terminal tail of $\mathrm{S}_{\mathrm{b} 4}$ therefore destabilizes the B-fold by $4.1 \mathrm{kcal} / \mathrm{mol}$ and observably populates the S-fold. Notably, the Rosetta energy of the design model of $S_{b 4}$ is virtually identical to that of $S_{b 3}$, even though $S_{b 3}$ actually has a much more stable S-fold (Fig. 4C). This reflects the influence of the antagonistic B-fold on the S-fold population in $\mathrm{S}_{\mathrm{b} 4}$. The antagonism of the B-fold is not reflected in the Rosetta energy of $S_{b 4}$, however, because the Relax protocol examines only a limited conformational space around the design model.

Design of $S_{b 5}$. We designed an L67R mutation in $S_{b 4}$ to destabilize the S-fold without changing the sequence of the embedded B-fold. The mutant is denoted as $S_{b 5}$. This was expected to shift the population to the B-fold. The energies of the computational models for $\mathrm{S}_{\mathrm{b} 4}$ and $S_{b 5}$ are shown in Fig. 4D. Note that the amino acid sequence for the 56 residue B-regions of $\mathrm{S}_{\mathrm{b} 4}$ and $\mathrm{S}_{\mathrm{b} 5}$ are the same as for $\mathrm{B}_{4}$.

Structural analysis of $\mathrm{S}_{\mathrm{b} 5}$. The $2 \mathrm{D}{ }^{1} \mathrm{H}_{-}{ }^{15} \mathrm{~N}$ HSQC spectrum of $\mathrm{S}_{\mathrm{b} 5}$ indicates that the $\mathrm{L} 67 \mathrm{R}$ mutation does indeed destabilize the S-fold, with the loss of S-type amide cross-peaks and the concurrent appearance of a new set of signals. Superposition of the spectrum with that of $B_{4}$ shows that the new signals in $S_{b 5}$ largely correspond with the spectrum of $B_{4}$ (Fig. S12). Thus, the L67R mutation shifts the equilibrium from the S-fold to the B-fold. The additional signals ( 25 30 ) in the central region of the HSQC spectrum that are not detected in $\mathrm{B}_{4}$ are presumably due to the disordered $\mathrm{C}$-terminal tail of $\mathrm{S}_{\mathrm{b} 5}$. In contrast to $\mathrm{S}_{\mathrm{b} 1}$, where the $\mathrm{C}$-terminal tail interacts with the B-fold extensively, there appears to be less interaction in $S_{b 5}$, as evidenced by fewer changes in chemical shifts or peak intensities in the B-region of $S_{b 5}$ compared with $B_{4}$. 
CD analysis of unfolding for $S_{b 5}$. The thermal unfolding profile of $S_{b 5}$ shows a low temperature transition with a midpoint $\sim 283^{\circ} \mathrm{K}$ and a major transition with a midpoint of $\sim 333^{\circ} \mathrm{K}$ (Fig. S4D). The NMR analysis indicates that the major transition is unfolding of the B-fold. Fitting the thermal denaturation data above $293^{\circ} \mathrm{K}$ to the Gibbs-Helmholtz equation shows the $\Delta \mathrm{G}_{\text {folding }}$ for the B-fold is $-5 \mathrm{kcal} / \mathrm{mol}$ at $298^{\circ} \mathrm{K}$ (Fig. 4B). Thus the L67R mutation in $\mathrm{S}_{\mathrm{b} 4}$ makes the B-fold highly favorable and the S-fold highly unfavorable $(>5 \mathrm{kcal} / \mathrm{mol}$ ) consistent with the change in population from mixed to B-fold observed by NMR. The large shift in S-fold population between $S_{b 4}(\sim 50 \%)$ and $S_{b 5}(\sim 0 \%)$ occurs with a moderate change in the Rosetta energy for the S-fold (Fig. 4D), however, due to the presence of competing alternative B-states. This is discussed further below.

\section{Discussion}

Five dimorphic sequence pairs were designed and stable structures were determined for 7 of the 10 proteins using NMR spectroscopy (Fig. 1). Two of the switches (one S- to Aswitch and one S- to B-switch) completely achieved the goal of populating the S-fold in the longer form and the G-fold in the shorter form. We initially assumed that mutations introduced to create compatibility with two folds would necessarily compromise native state interactions in one or both of the folds. The surprising conclusion, however, is that for one S- to A-switch, and three of four S- to B-switches it was possible to design one sequence that is compatible with native state interactions for both folds. In all these cases the calculated energy of the S-fold was near the wild type sequence. This was true even though many mutations were introduced to create compatibility. It is important to understand, however, that Rosetta Relax evaluates native state interactions in the vicinity of the starting structure. The $\Delta \mathrm{G}_{\text {folding }}$ of dimorphic 
proteins will also be strongly influenced by non-native states that the Relax protocol is not evaluating.

Engineering stability of an antagonistic, embedded fold necessarily destabilizes the longer fold even when native-state interactions are not compromised. Consequently, three basic structural transitions dictate the behavior of a dimorphic protein. To understand these transitions, it is useful to divide the structure of the longer protein into the sequence corresponding to the 56 residue embedded fold (part 1) and the remaining sequence (part 2). Both parts are ordered in the S-fold. When part 1 switches into a G-fold, however, part 2 unfolds. The conformations of part 1 are denoted s1 (S-conformation), g1 (A- or Bconformation), and u1 (unfolded conformation). The conformations of part 2 are denoted s2 (S-conformation) and u2 (unfolded conformation). Consider the equilibria:

s1-s2 $\rightleftarrows \mathrm{u} 1-\mathrm{u} 2 \rightleftarrows \mathrm{g} 1-\mathrm{u} 2 \quad$ Transitions of the longer dimorphic sequence

$\mathrm{g} 1 \rightleftarrows \mathrm{u} 1 \rightleftarrows \mathrm{s} 1 \quad$ Transitions of the shorter sequence.

In an idealized dimorphic protein, the energy from native interactions in both S- and Gfolds would be equivalent to native interactions in the two natural proteins. If we then assume that part 2 only interacts with s1 to form s1-s2 or with solvent in g1-u2 and u1-u2 forms, then we can predict the populations of all the species in the switch from the equilibrium constants for the S-fold $\left(\mathrm{K}_{\mathrm{S}}\right)$ and the A-fold $\left(\mathrm{K}_{\mathrm{A}}\right)$. These are calculated using the $\Delta \mathrm{G}_{\text {folding }}$ of the wild type proteins (Fig. 4) and the Gibbs equation $\Delta G=-R T$ In (K). For example, the expected populations in an idealized switch from the S- to the A-fold would be:

$$
\begin{aligned}
& \mathrm{s} 1-\mathrm{s} 2 \stackrel{\mathrm{Ks}}{\rightleftarrows} \quad \mathrm{u} 1-\mathrm{u} 2 \quad \mathrm{~K}_{\mathrm{s}}=[\mathrm{s} 1-\mathrm{s} 2] /[\mathrm{u} 1-\mathrm{u} 2]=1.4 \times 10^{6} \quad \text { (unfolding of } \mathrm{S} \text { ) } \\
& \mathrm{K}_{\mathrm{A}} \\
& \text { a1-u2 } \rightleftarrows \quad \text { u1-u2 } \quad K_{A}=[\mathrm{a} 1-\mathrm{u} 2] /[\mathrm{u} 1-\mathrm{u} 2]=1.1 \times 10^{4} \quad \text { (unfolding of } A \text { ) }
\end{aligned}
$$


s1-s2 $\stackrel{\mathrm{K}_{\mathrm{S}-\mathrm{A}}}{\rightleftarrows}$ a1-u2 $\quad \mathrm{K}_{\mathrm{S}-\mathrm{A}}=[\mathrm{s} 1-\mathrm{s} 2] /[\mathrm{a} 1-\mathrm{u} 2]=\mathrm{K}_{\mathrm{S}} / \mathrm{K}_{\mathrm{A}}=127 \quad$ (switching of $\mathrm{S}$ and $\mathrm{A}$ )

Rules for switches emerge from examining deviations from idealized behavior. Each of the five dimorphic sequences were assessed for how well the experimental structure matches the design and how well the switching energetics match the idealized case. We observe behaviors ranging from near ideal to large deviations from ideal, but surprisingly, most deviations do not appear to result from compromised native interactions in S- and G-folds. Rather, deviations appear to arise from promiscuous interactions of $\mathrm{u} 2$ with alternative folds of part 1 . That is, the assumption that $\mathrm{u} 2$ interacts only with $\mathrm{s} 1$ to form an S-fold or with solvent in an unfolded state is invalid. In fact, s2 forms alternative interactions with the G-fold that compete with formation of the S-fold.

Case $1, S_{a 1}$ to $A_{1}$ switch: Part 1 in this switch comprises residues $11-66$ and part 2 residues 1-10 and 67-95. The experimental and designed structures of $S_{a 1}$ match (Fig. 2), Rosetta energies are similar, and the observed $\Delta \mathrm{G}_{\text {folding }}$ of $\mathrm{S}_{\mathrm{a} 1}$ is $-7.5 \mathrm{kcal} / \mathrm{mol}$, compared to an idealized value of $-8.5 \mathrm{kcal} / \mathrm{mol}$. Likewise, the 56 amino acid $A_{1}$ protein has a stable structure that closely matches the designed model. This example comes closest to an idealized case. This switching behavior occurs because the S-fold is considerably more stable than the embedded, antagonistic A-fold and the equilibrium strongly favors $S$ in the longer protein (Fig. 4C).

Case 2, $S_{b 1}$ to $B_{1}$ switch: Part 1 in this switch comprises residues 5-60 and part 2 residues 1-4 and 61-95. The designed and experimental structures of $B_{1}$ match quite well in this case, but those of $S_{b 1}$ do not (Fig. 5). In fact, $S_{b 1}$ populates a B-like fold even though the Rosetta energy of the $S_{b 1}$ design model is only a little less than native $S$ protein (Fig. 4D). Close examination of the ensemble of NMR structures for $S_{b 1}$, shows that part 2 of the protein has 
propensity for S-type structures even when part 1 has a B-fold. Thus, the observed conformational ensemble can be denoted g1-s2. This ensemble is populated because stability of the internal g1-state compromises the stability of the s1-s2-fold and results in promiscuous interactions of $\mathrm{s} 2$ with g1. The overall $\Delta \mathrm{G}_{\text {folding }}$ for the ensemble of B-like folds in $\mathrm{S}_{\mathrm{b} 1}$ is -1.1 $\mathrm{kcal} / \mathrm{mol}$. Since no S-fold is observed in $\mathrm{S}_{\mathrm{b} 1}$, this means that the $\Delta \mathrm{G}_{\text {folding }}$ for the $\mathrm{S}$-fold is $>1.1$ $\mathrm{kcal} / \mathrm{mol}$ (Fig. 4C).

Case 3, $S_{b 2}$ to $B_{2}$ switch: To generate a second version of this switch, we introduced 13 mutations into the $S_{b 1}$ sequence. The mutations result in a switch from a B-fold into an S-fold and the designed and experimental structures of $\mathrm{S}_{\mathrm{b} 2}$ roughly match (Fig. S6). The observed $\Delta \mathrm{G}_{\text {folding }}$ of $\mathrm{S}_{\mathrm{b} 2}$ is $-4.0 \mathrm{kcal} / \mathrm{mol}$. The switch from B- to $\mathrm{S}$-folds does not appear to arise from improved native interactions in the S-fold, however. In fact, the Rosetta energy of the $\mathrm{S}_{\mathrm{b} 2}$ design model is almost identical to the $S_{b 1}$ design model (Fig. 4D). Rather, the B- to S-switch results from decreased stability of the antagonistic, embedded B-fold in $S_{b 2}$.

Case 4, $S_{b 3}$ to $B_{3}$ switch: Part 1 in this switch comprises residues 1-56 and part 2 residues 57-87. The designed and experimental structures roughly match (Fig. 6). S S populates an S-fold, although deviations exist in loops. The Rosetta energy of the $S_{b 3}$ design model is very similar to that of the natural sequence (Fig. 4D). The observed $\Delta \mathrm{G}_{\text {folding }}$ of the S-fold is only -3.5 $\mathrm{kcal} / \mathrm{mol}$, however, and shows the influence of the antagonistic B-fold on S-fold stability. $\mathrm{B}_{3}$ primarily populates a B-fold, but similarly its low stability $(-1.2 \mathrm{kcal} / \mathrm{mol})$ may indicate some propensity for the antagonistic S-conformation.

Case 5, $S_{b 4}$ and $S_{b 5}$ to $B_{4}$ switch: The $Y 5 L$ mutation introduced into $S_{b 3}$ results in simultaneous population of multiple, folded conformational states. This appears to arise from 
slightly compromised native interactions in the S-fold and increased stability of the antagonistic, embedded B-fold. The 56 amino acid $\mathrm{B}_{4}$ protein has a B-fold that matches the designed model. The $\Delta \mathrm{G}_{\text {folding }}$ of $\mathrm{B}_{4}$ is $-4.1 \mathrm{kcal} / \mathrm{mol}$ compared to $-6.6 \mathrm{kcal} / \mathrm{mol}$ for the natural $\mathrm{G}_{\mathrm{B}}$ protein. This is an example in which good computational design produces a complex result. The complexity appears to arise because the stability of the embedded B-fold and the longer S-folds are similar and antagonistic. This causes more than one fold to be populated and the observed stability of the $S$-fold to be lower than predicted based on its REU value. $S_{b 4}$ is thus at a critical point in switching between the S- and B-folds. Consequently, single substitution mutations in $\mathrm{S}_{\mathrm{b} 4}$ can produce either a stable S-fold or a stable B-fold (Fig. 8).

Several general rules for engineering switches emerge from this study. First, it is possible to design one sequence with good native state interactions in two folds. If this condition is met, then the two main factors determining fold populations are the stability of the embedded G-fold relative to the S-fold and the conformational propensities of the ends that are generated in the switch to the embedded fold. The higher the stability of the embedded fold relative to the larger fold, the more the ends are populated, and the more the structures deviate from design (e.g. $S_{b_{1}}$ ). Thus, the ends generated in a switch are a double-edged sword. They are a repository of switching energy to drive the G-fold to the S-fold but also can contribute energy to switch to other states. Finally, successful design of a dimorphic sequence creates a critical state in which conformation is extremely sensitive to small perturbations anywhere in the sequence. Thus, as in other complex systems, a small change may have a "butterfly effect" on how the folds are populated (Fig. 8).

Rules for engineering switches also highlight several well-established principles of protein folding. First, the stability of the non-native state, though less predictable, contributes to 
overall stability as much as the native state (53-60). Second, the effect of the appended ends is often denaturing. Ends can be generally denaturing by forming non-specific backbone hydrogen bonds and hydrophobic interactions in the unfolded state ensemble, but promiscuous interactions of structured end fragments may also result in alternative structures (61). This study also shows a surprising attribute of the folding code: It is not difficult to engineer sequences that are compatible with native interactions in more than one fold. This is consistent with the existence of natural dimorphic proteins, but the fact remains that most natural protein sequences populate only one fold (62). We suggest that evolutionary pressure to avoid critical states typically causes proteins to evolve toward a single native state. Thus, two-state behavior may result from nature's negative design rather than being an inherent property of the protein

code (63-65). Proteins generally evolve toward two-state behavior because dimorphic sequences can generate promiscuous interactions that destabilize them, compromise their function, and may be pathological.

\section{Materials and Methods}

Mutagenesis, protein expression and purification. Mutagenesis was carried out using Q $5^{\circ}$ Site-Directed Mutagenesis Kits (NEB). $\mathrm{G}_{A}$ and $\mathrm{G}_{B}$ variants were cloned into a vector (pAYRGL) encoding the sequence:

MEEAVDANSLAQAKEAAIKELKQYGIGDKYIKLINNAKTVEGVESLKNE ILKALPTEGSGEEDKQYRGL-

as an $\mathrm{N}$-terminal fusion domain (39). The resulting fusion proteins were purified using a second generation of the affinity-cleavage tag system used previous to purify switch proteins $(8,66)$.

The second generation tag (YRGL-tag) results in high-level soluble expression of the switch proteins and also enables capture of the fusion protein by binding tightly to an immobilized 
processing protease via the C-terminal EEDQYRGL sequence. The addition of $100 \mu \mathrm{M}$ imidazole activates an immobilized, imidazole-activated protease (Im-Prot) and releases the purified switch protein from the Im-Prot media (Potomac Affinity Proteins). The purified protein was then concentrated to 0.2 to $0.3 \mathrm{mM}$, as required for NMR analysis. The purification system is described in detail in Supplemental methods and is available from Potomac Affinity Proteins.

Circular Dichroism (CD). CD measurements were performed in $100 \mathrm{mM} \mathrm{KPO}_{4}, \mathrm{pH} 7.2$ with a Jasco spectropolarimeter, model J-1100 with a Peltier temperature controller. Quartz cells with path lengths of $0.1 \mathrm{~cm}$ and $1 \mathrm{~cm}$ were used for protein concentrations of 3 and $30 \mu \mathrm{M}$, respectively. The ellipticity results were expressed as mean residue ellipticity, $[\theta], \operatorname{deg} \mathrm{cm}^{2}$ $\mathrm{dmol}^{-1}$. Ellipticities at $222 \mathrm{~nm}$ were continuously monitored at a scanning rate $0.5 \mathrm{deg} / \mathrm{min}$. Reversibility of denaturation was confirmed by comparing the CD spectra at $293^{\circ} \mathrm{K}$ before melting and after heating to $373^{\circ} \mathrm{K}$ and cooling to $293^{\circ} \mathrm{K}$.

NMR Spectroscopy. Isotope-labeled samples were prepared at 0.2-0.3 mM concentrations in $100 \mathrm{mM}$ potassium phosphate buffer $(\mathrm{pH} 7.0)$ containing $5 \% \mathrm{D}_{2} \mathrm{O}$. NMR spectra were collected on Bruker AVANCE III 600 and $900 \mathrm{MHz}$ spectrometers fitted with Zgradient ${ }^{1} \mathrm{H} /{ }^{13} \mathrm{C} /{ }^{15} \mathrm{~N}$ triple resonance cryoprobes. Standard double and triple resonance experiments ( $\mathrm{HNCACB}, \mathrm{CBCA}(\mathrm{CO}) \mathrm{NH}, \mathrm{HNCO}, \mathrm{HN}(\mathrm{CA}) \mathrm{CO}$, and $\mathrm{HNHA}$ ) were utilized to determine main chain NMR assignments. Inter-proton distances were obtained from $3 \mathrm{D}^{15} \mathrm{~N}$-edited NOESY and $3 D^{13} \mathrm{C}$-edited NOESY spectra with a mixing time of $150 \mathrm{~ms}$. NmrPipe (67) was used for data processing and analysis was done with Sparky (68). Two-dimensional $\left\{{ }^{1} \mathrm{H}\right\}-{ }^{15} \mathrm{~N}$ steady state heteronuclear NOE experiments were acquired with a $5 \mathrm{~s}$ relaxation delay between experiments. Chemical shift perturbations between $\mathrm{B}_{1}$ and $\mathrm{S}_{\mathrm{b} 1}$ were calculated using $\Delta \delta_{\text {total }}=\left(\left(W_{H} \Delta \delta_{H}\right)^{2}+\left(W_{N} \Delta \delta N\right)^{2}\right)^{1 / 2}$, where $\Delta \delta_{H}$ and $\Delta \delta_{N}$ represent ${ }^{1} H$ and ${ }^{15} \mathrm{~N}$ chemical shift 
changes, respectively. For PRE experiments on $S_{b 1}$, single-site cysteine mutant samples were incubated with 10 equivalents of MTSL ((1-oxyl-2,2,5,5-tetramethylpyrroline-3-methyl) methanethiosulfonate, Santa Cruz Biotechnology) at $25^{\circ} \mathrm{C}$ for 1 hour and completion of labeling was confirmed by MALDI mass spectrometry. Control samples were reduced with 10 equivalents of sodium ascorbate. Backbone amide peak intensities of the oxidized and reduced states were analyzed using Sparky. Three-dimensional structures were calculated with CS-Rosetta3.2 using experimental backbone ${ }^{15} \mathrm{~N},{ }^{1} \mathrm{H}_{\mathrm{N}},{ }^{1} \mathrm{H} \alpha{ }^{13} \mathrm{C} \alpha,{ }^{13} \mathrm{C} \beta$, and ${ }^{13} \mathrm{CO}$ chemical shift restraints and were either validated by comparison with experimental backbone NOE patterns $\left(A_{1}, B_{1}, B_{4}, S_{b 1}\right)$ or directly employed interproton NOEs $\left(\mathrm{S}_{\mathrm{a} 1}, \mathrm{~S}_{\mathrm{b} 2}\right)$ or PREs $\left(\mathrm{S}_{\mathrm{b}_{1}}\right)$ as additional restraints. One thousand CS-Rosetta structures were calculated from which the 10 lowest energy structures were chosen. For $\mathrm{S}_{\mathrm{b} 3}$, CS-Rosetta failed to converge to a unique low energy topology, producing an approximately even mixture of S- and B-type folds despite the chemical shifts and NOE pattern indicating an S-fold. In this case, CNS1.1 (69) was employed to determine the structure as described previously (39), including backbone dihedral restraints from chemical shift data using TALOS (70). Protein structures were displayed and analyzed utilizing PROCHECK-NMR (71), MOLMOL (72) and PyMol (Schrodinger) (38).

\section{Acknowledgments}

This work was supported by National Institutes of Health Grant GM62154 (to PB and JO) and 5R44GM126676 (to PB). The NMR facility is supported by the University of Maryland, the National Institute of Standards and Technology, and a grant from the W. M. Keck Foundation. We would also like to thank Dr. Nese Sari for her thoughtful comments. Mention of commercial products does not imply recommendation or endorsement by NIST. 


\section{References}

1. X. I. Ambroggio, B. Kuhlman, Design of protein conformational switches. Curr Opin Struct Biol 16, 525-530 (2006).

2. P. N. Bryan, J. Orban, Proteins that switch folds. Curr. Opin. Struct. Biol. 20, 482-488 (2010).

3. A. F. Dishman et al., Evolution of fold switching in a metamorphic protein. Science 371, 86-90 (2021).

4. K. Y. Wei et al., Computational design of closely related proteins that adopt two welldefined but structurally divergent folds. Proc Natl Acad Sci U S A 117, 7208-7215 (2020).

5. W. J. Anderson, L. O. Van Dorn, W. M. Ingram, M. H. Cordes, Evolutionary bridges to new protein folds: design of C-terminal Cro protein chameleon sequences. Protein Eng Des Sel 24, 765-771 (2011).

6. B. M. Burmann et al., An $\alpha$ helix to $\beta$ barrel domain switch transforms the transcription factor RfaH into a translation factor. Cell 150, 291-303 (2012).

7. P. Kulkarni et al., Structural metamorphism and polymorphism in proteins on the brink of thermodynamic stability. Protein Sci 27, 1557-1567 (2018).

8. P. A. Alexander, Y. He, Y. Chen, J. Orban, P. N. Bryan, A minimal sequence code for switching protein structure and function. Proc Natl Acad Sci U S A 106, 21149-21154 (2009).

9. Y. He, Y. Chen, P. A. Alexander, P. N. Bryan, J. Orban, Mutational tipping points for switching protein folds and functions. Structure 20, 283-291 (2012).

10. R. Day, D. A. Beck, R. S. Armen, V. Daggett, A consensus view of fold space: combining SCOP, CATH, and the Dali Domain Dictionary. Protein Sci 12, 2150-2160 (2003).

11. S. Rackovsky, Nonlinearities in protein space limit the utility of informatics in protein biophysics. Proteins 83, 1923-1928 (2015).

12. S. H. Chen, J. Meller, R. Elber, Comprehensive analysis of sequences of a protein switch. Protein Sci 25, 135-146 (2016).

13. W. Li, L. N. Kinch, P. A. Karplus, N. V. Grishin, ChSeq: A database of chameleon sequences. Protein Sci 24, 1075-1086 (2015).

14. P. G. Wolynes, Evolution, energy landscapes and the paradoxes of protein folding. Biochimie 119, 218-230 (2015).

15. C. Holzgräfe, S. Wallin, Smooth functional transition along a mutational pathway with an abrupt protein fold switch. Biophys J 107, 1217-1225 (2014).

16. H. A. Scheraga, S. Rackovsky, Homolog detection using global sequence properties suggests an alternate view of structural encoding in protein sequences. Proc Natl Acad Sci U S A 111, 5225-5229 (2014).

17. J. H. Ha, S. N. Loh, Protein conformational switches: from nature to design. Chemistry 18, 7984-7999 (2012).

18. I. Yadid, N. Kirshenbaum, M. Sharon, O. Dym, D. S. Tawfik, Metamorphic proteins mediate evolutionary transitions of structure. Proc Natl Acad Sci U S A 107, 7287-7292 (2010).

19. O. Lichtarge, A. Wilkins, Evolution: a guide to perturb protein function and networks. Curr Opin Struct Biol 20, 351-359 (2010).

20. N. J. Rollins et al., Inferring protein 3D structure from deep mutation scans. Nat Genet 51, 1170-1176 (2019). 
21. T. Sikosek, H. S. Chan, E. Bornberg-Bauer, Escape from Adaptive Conflict follows from weak functional trade-offs and mutational robustness. Proc Natl Acad Sci U S A 109, 14888-14893 (2012).

22. N. Chen, M. Das, A. LiWang, L. P. Wang, Sequence-Based Prediction of Metamorphic Behavior in Proteins. Biophys J 119, 1380-1390 (2020).

23. L. L. Porter, L. L. Looger, Extant fold-switching proteins are widespread. Proc Natl Acad Sci U S A 115, 5968-5973 (2018).

24. J. T. Bedford, J. Poutsma, N. Diawara, L. H. Greene, The nature of persistent interactions in two model $\beta$-grasp proteins reveals the advantage of symmetry in stability. Journal of Computational Chemistry 42, 600-607 (2021).

25. C. Falkenberg, L. Bjorck, B. Akerstrom, Localization of the binding site for streptococcal protein $\mathrm{G}$ on human serum albumin. Identification of a 5.5-kilodalton protein $\mathrm{G}$ binding albumin fragment. Biochemistry 31, 1451-1457 (1992).

26. I. M. Frick et al., Convergent evolution among immunoglobulin G-binding bacterial proteins. Proc Natl Acad Sci U S A 89, 8532-8536 (1992).

27. E. B. Myhre, G. Kronvall, Heterogeneity of nonimmune immunoglobulin Fc reactivity among gram-positive cocci: description of three major types of receptors for human immunoglobulin G. Infect. Immun. 17, 475-482 (1977).

28. K. J. Reis, E. M. Ayoub, M. D. P. Boyle, Streptococcal Fc receptors. II. Comparison of the reactivity of a receptor from a group $\mathrm{C}$ streptococcus with staphylococcal protein $\mathrm{A}$. J. Immunol. 132, 3098-3102 (1984).

29. M. O. Lindberg, E. Haglund, I. A. Hubner, E. I. Shakhnovich, M. Oliveberg, Identification of the minimal protein-folding nucleus through loop-entropy perturbations. Proc Natl Acad Sci U S A 103, 4083-4088 (2006).

30. E. Haglund, M. O. Lindberg, M. Oliveberg, Changes of protein folding pathways by circular permutation. Overlapping nuclei promote global cooperativity. J Biol Chem 283, 27904-27915 (2008).

31. E. Haglund et al., The HD-exchange motions of ribosomal protein $\mathrm{S} 6$ are insensitive to reversal of the protein-folding pathway. Proc Natl Acad Sci U S A 106, 21619-21624 (2009).

32. E. Haglund et al., Trimming down a protein structure to its bare foldons: spatial organization of the cooperative unit. J Biol Chem 287, 2731-2738 (2012).

33. M. Lindahl et al., Crystal structure of the ribosomal protein S6 from Thermus thermophilus. Embo j 13, 1249-1254 (1994).

34. M. Steinegger et al., HH-suite3 for fast remote homology detection and deep protein annotation. BMC Bioinformatics 20, 473 (2019).

35. A. Leaver-Fay et al., ROSETTA3: an object-oriented software suite for the simulation and design of macromolecules. Methods Enzymol 487, 545-574 (2011).

36. P. A. Alexander, D. A. Rozak, J. Orban, P. N. Bryan, Directed evolution of highly homologous proteins with different folds by phage display: implications for the protein folding code. Biochemistry 44, 14045-14054 (2005).

37. P. A. Alexander, Y. He, Y. Chen, J. Orban, P. N. Bryan, The design and characterization of two proteins with $88 \%$ sequence identity but different structure and function. Proc NatI Acad Sci U S A 104, 11963-11968 (2007).

38. W. L. Delano (2002) The PyMOL Molecular Graphics System. (DeLano Scientific, San Carlos, CA). 
39. Y. He et al., Structure, dynamics, and stability variation in bacterial albumin binding modules: implications for species specificity. Biochemistry 45, 10102-10109 (2006).

40. Y. Shen et al., De novo structure generation using chemical shifts for proteins with highsequence identity but different folds. Protein Sci. 19, 349-356 (2010).

41. W. J. Becktel, J. A. Schellman, Protein stability curves. Biopolymers 26, 1859-1877 (1987).

42. D. A. Rozak, J. Orban, P. N. Bryan, G148-GA3: a streptococcal virulence module with atypical thermodynamics of folding optimally binds human serum albumin at physiological temperatures. Biochim Biophys Acta 1753, 226-233 (2005).

43. Y. He, Y. Chen, D. A. Rozak, P. N. Bryan, J. Orban, An artificially evolved albumin binding module facilitates chemical shift epitope mapping of GA domain interactions with phylogenetically diverse albumins. Protein Sci 16, 1490-1494 (2007).

44. P. Alexander, S. Fahnestock, T. Lee, J. Orban, P. Bryan, Thermodynamic analysis of the folding of the Streptococcal Protein G IgG-binding domains B1 and B2: why small proteins tend to have high denaturation temperatures. Biochemistry 31, 3597-3603 (1992).

45. E. L. McCallister, E. Alm, D. Baker, Critical role of beta-hairpin formation in protein G folding. Nat Struct Biol 7, 669-673 (2000).

46. D. Khare et al., $\mathrm{pK}_{\mathrm{a}}$ measurements from nuclear magnetic resonance for the B1 and B2 immunoglobulin G-binding domains of protein G: Comparison with calculated values for nuclear magnetic resonance and x-ray structures. Biochemistry 36, 3580-3589 (1997).

47. P. Alexander, J. Orban, P. Bryan, Kinetic analysis of folding and unfolding the 56 amino acid IgG-binding domain of Streptococcal Protein G. Biochemistry 31, 7243-7248 (1992).

48. F. J. Blanco, G. Rivas, L. Serrano, A short linear peptide that folds into a native stable bhairpin in aqueous solution. Nature Struct. Biol. 1, 584-590 (1994).

49. C. A. Orengo, J. M. Thornton, Alpha plus beta folds revisited: some favoured motifs. Structure 1, 105-120 (1993).

50. T. D. Gallagher, G. Gilliland, L. Wang, P. Bryan, The prosegment-subtilisin BPN' complex: crystal structure of a specific foldase. Structure 3, 907-914 (1995).

51. M. A. Tangrea, P. N. Bryan, N. Sari, J. Orban, Solution Structure of the Pro-hormone Convertase 1 Pro-domain from Mus musculus. J Mol Biol 320, 801-812 (2002).

52. Y. He et al., Solution NMR structure of a sheddase inhibitor prodomain from the malarial parasite Plasmodium falciparum. Proteins 80, 2810-2817 (2012).

53. H. J. Dyson, P. E. Wright, Equilibrium NMR studies of unfolded and partially folded proteins. Nat Struct Biol 5 Suppl, 499-503 (1998).

54. H. Fu, G. Grimsley, J. M. Scholtz, C. N. Pace, Increasing protein stability: importance of DeltaC(p) and the denatured state. Protein Sci 19, 1044-1052 (2010).

55. V. L. Arcus, S. Vuilleumier, S. M. V. Freund, M. Bycroft, A. R. Fersht, Toward solving the folding pathway of barnase: The complete backbone ${ }^{13} \mathrm{C},{ }^{15} \mathrm{~N}$, and ${ }^{1} \mathrm{H}$ NMR assignments of its pH-denatured state. Proc. Natl. Acad. Sci. USA 91, 9412-9416 (1994).

56. K. A. Dill, D. Shortle, Denatured states of proteins. Annu. Rev. Biochem. 60, 795-825 (1991).

57. Q. Yi, M. L. Scalley-Kim, E. J. Alm, D. Baker, NMR characterization of residual structure in the denatured state of protein L. J Mol Biol 299, 1341-1351. (2000). 
58. A. Morrone et al., The denatured state dictates the topology of two proteins with almost identical sequence but different native structure and function. J Biol Chem 286, 3863-3872 (2011).

59. P. L. Clark, K. W. Plaxco, T. R. Sosnick, Water as a Good Solvent for Unfolded Proteins: Folding and Collapse are Fundamentally Different. J Mol Biol 432, 2882-2889 (2020).

60. M. A. Bowman et al., Properties of protein unfolded states suggest broad selection for expanded conformational ensembles. Proc Natl Acad Sci U S A 117, 23356-23364 (2020).

61. M. J. Bennett, M. R. Sawaya, D. Eisenberg, Deposition diseases and 3D domain swapping. Structure 14, 811-824 (2006).

62. C. B. Anfinsen, Principles that govern the folding of protein chains. Science 181, 223-230 (1973).

63. A. I. Gilson, A. Marshall-Christensen, J. M. Choi, E. I. Shakhnovich, The Role of Evolutionary Selection in the Dynamics of Protein Structure Evolution. Biophys $J$ 112, 1350-1365 (2017).

64. T. N. Starr, J. W. Thornton, Epistasis in protein evolution. Protein Sci 25, 1204-1218 (2016).

65. J. Hoffmann, J. O. Wrabl, V. J. Hilser, The role of negative selection in protein evolution revealed through the energetics of the native state ensemble. Proteins 84, 435-447 (2016).

66. B. Ruan, K. E. Fisher, P. A. Alexander, V. Doroshko, P. N. Bryan, Engineering subtilisin into a fluoride-triggered processing protease useful for one-step protein purification. Biochemistry 43, 14539-14546 (2004).

67. F. Delaglio et al., NMRPipe: a multidimensional spectral processing system based on UNIX pipes. J. Biomol. NMR 6, 277-293 (1995).

68. T. D. Goddard, D. G. Kneller (2004) SPARKY 3. (University of California San Francisco).

69. A. T. Brunger et al., Crystallography \& NMR system: A new software suite for macromolecular structure determination. Acta Crystallogr. D (Biol. Crystallogr.) 54, 905921 (1998).

70. G. Cornilescu, F. Delaglio, A. Bax, Protein backbone angle restraints from searching a database for chemical shift and sequence homology. J. Biomol. NMR 13, 289-302 (1999).

71. R. A. Laskowski, J. A. Rullmann, M. W. MacArthur, R. Kaptein, J. M. Thornton, AQUA and PROCHECK-NMR: Programs for checking the quality of protein structures solved by NMR. J. Biomol. NMR 8, 477-486 (1996).

72. R. Koradi, M. Billeter, K. Wuthrich, MOLMOL: a program for display and analysis of macromolecular structures. J Mol Graph 14, 51-55 (1996). 


\section{Figures}

S / A Design

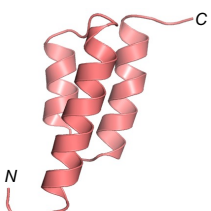

$A_{1}$

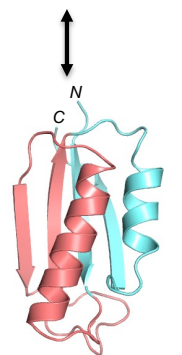

$\mathbf{S}_{\mathrm{a} 1}$

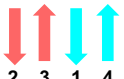

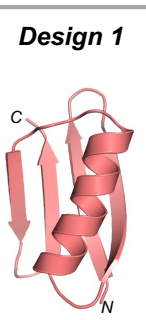

$\mathrm{B}_{1}$
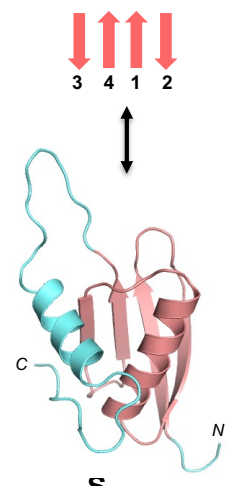

$\mathrm{s}_{\mathrm{b} 1}$

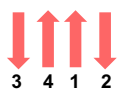

\section{S / B Designs}

Design 2

Design 3

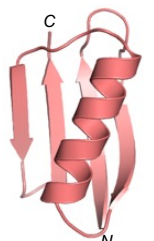

$\mathrm{B}_{3}$

$B_{2}$
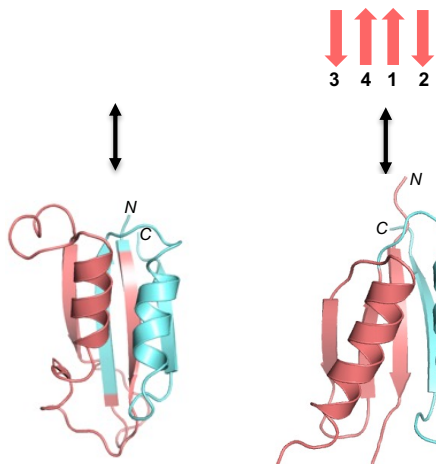

$\mathrm{s}_{\mathrm{b} 2}$

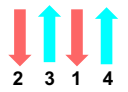

Design 4

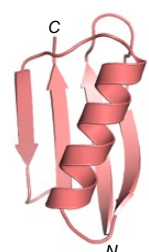

$\mathrm{B}_{4}$

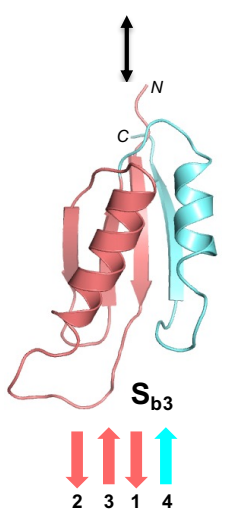

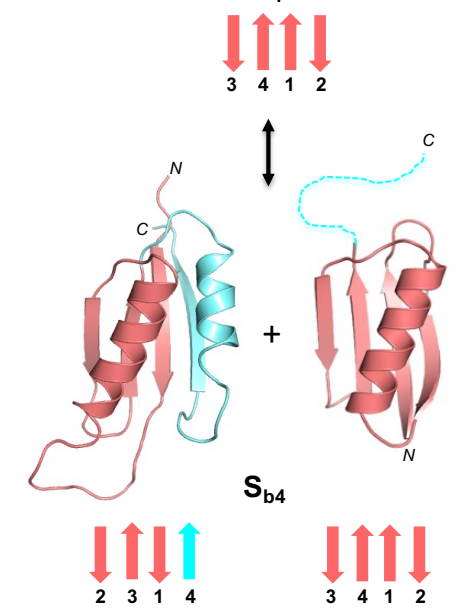

Figure 1: Summary of designed dimorphic proteins. Cartoon structures of five pairs of dimorphic proteins are depicted based on the NMR structures of $A_{1}, B_{1}, B_{4}, S_{a 1}, S_{b 1}, S_{b 2}$, and $S_{b 3}$ that are determined here. The common sequence in each pair is in red. The extra amino acids in the longer sequences are in cyan. The arrows at the bottom of $\beta$-sheet containing structures show the topology of $\beta$-strands. 
A

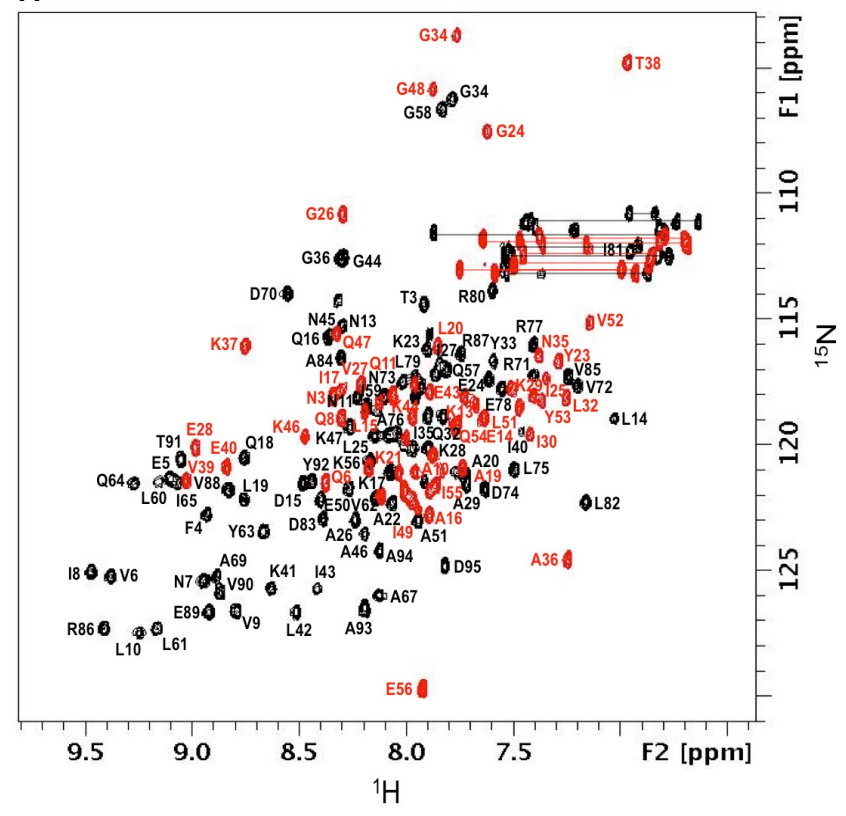

B

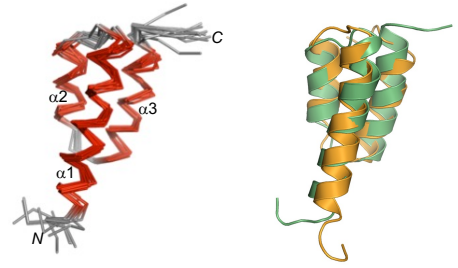

c
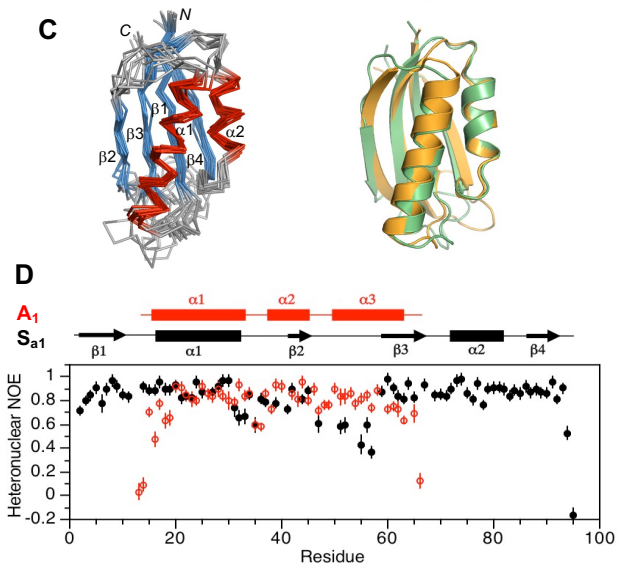

Figure 2: Structure and dynamics of $A_{1}$ and $S_{a 1}$. (A) Overlaid two dimensional ${ }^{1} \mathrm{H}^{-15} \mathrm{~N} H S Q C$ spectra of $S_{a 1}$ (black) and $A_{1}$ (red) with backbone amide assignments. Spectra were recorded at $298^{\circ} \mathrm{K}$ and $278^{\circ} \mathrm{K}$, respectively. (B) Ensemble of 10 lowest energy CS-Rosetta structures for $A_{1}$ (left panel). Superposition of the $A_{1}$ structure (green) with the parent $G_{A}$ fold (orange) (right panel). (C) Ensemble of 10 lowest energy CS-Rosetta structures for $\mathrm{S}_{\mathrm{a} 1}$ (left panel). Superposition of $S_{a 1}$ (green) with the parent $S 6$ fold (orange) (right panel). (D) Backbone dynamics in designed proteins. Plot of $\left\{{ }^{1} \mathrm{H}\right\}-{ }^{15} \mathrm{~N}$ steady state heteronuclear NOE values at 600 $\mathrm{MHz}$ versus residue for $\mathrm{A}_{1}$ (red) and for $\mathrm{S}_{\mathrm{a} 1}$ (black). Error bars indicate $\pm 1 \mathrm{SD}$. 
A
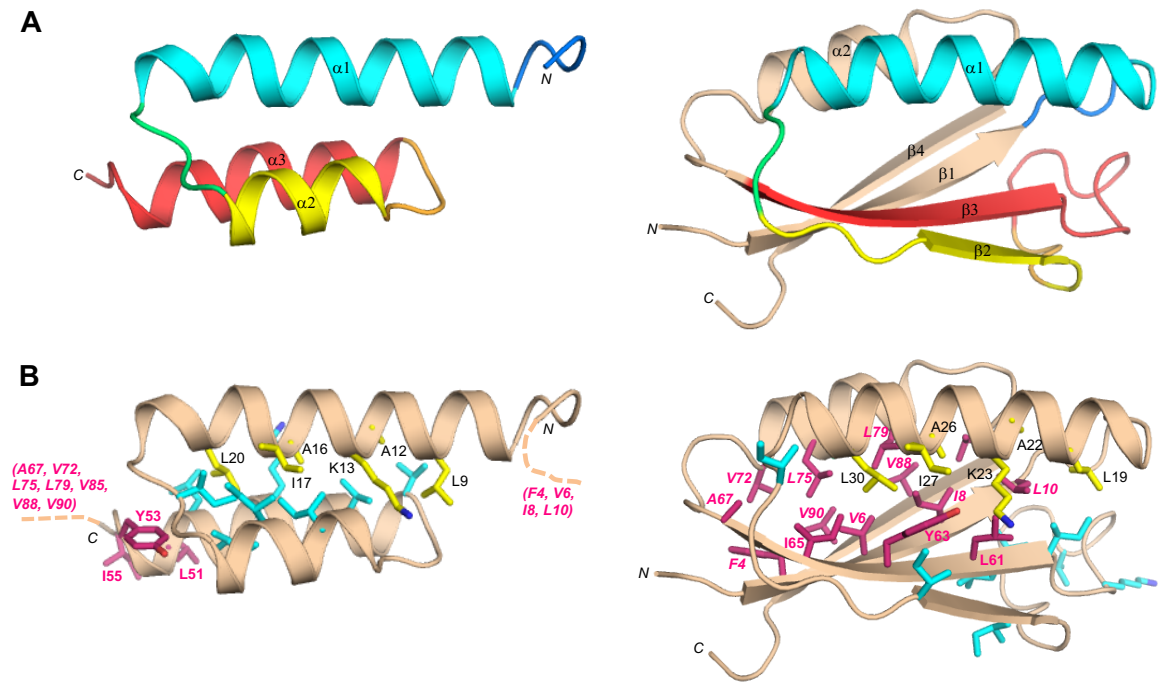

Figure 3: Structural differences between the sequence identical regions of $A_{1}$ and $S_{a 1}$. (A) Main chain comparisons. (Left panel) CS-Rosetta structure of $A_{1}$ with color coding for secondary structured elements. (Right panel) Corresponding color-coded regions mapped onto the CSRosetta structure of $S_{a 1}$, illustrating changes in backbone conformation. Regions outside the 56 amino acid sequence of $A_{1}$ are shown in wheat. (B) Side chain comparisons. (Left panel) Residues contributing to the core of $A_{1}$ from the $\alpha 1$-helix (yellow), and from other regions (cyan). The non- $\alpha 1$ core residues from $S_{a 1}$ (pink) do not overlap with the $A_{1}$ core (see text for further details). (Right panel) Residues contributing to the core of $S_{a 1}$ from the $\alpha 1$-helix (yellow), and most of the other participating core residues (pink). The non- $\alpha 1$ core residues from $A_{1}$ are also shown (cyan), highlighting the low degree of overlap. 
A

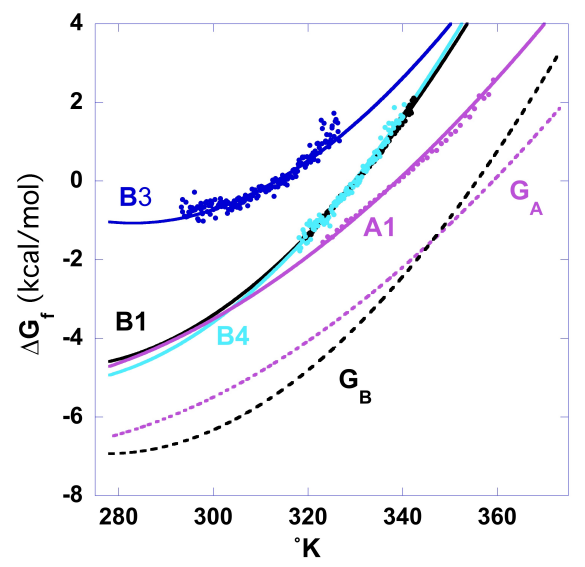

C

$\Delta G_{f}$

$A_{1}(-4.0)$

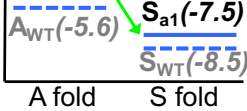

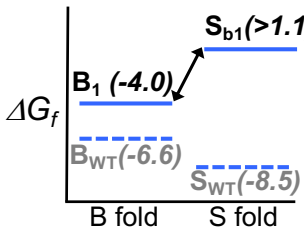

$\Delta G_{f} \mid$\begin{tabular}{ll}
$\frac{B_{3}(-1.2)}{B_{W T}(-\overline{6} . \overline{6})}$ & \\
\hline $\bar{S}_{\text {WT }}(-\overline{8}(-\overline{5})$ \\
B fold & $S$ fold
\end{tabular}
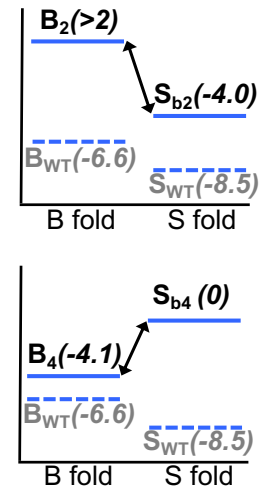

B

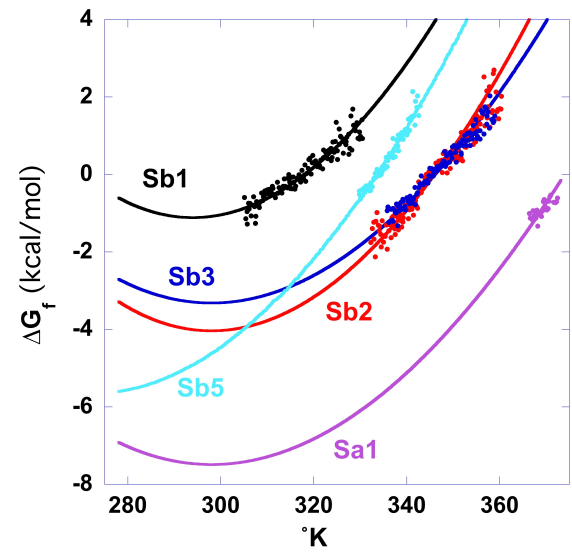

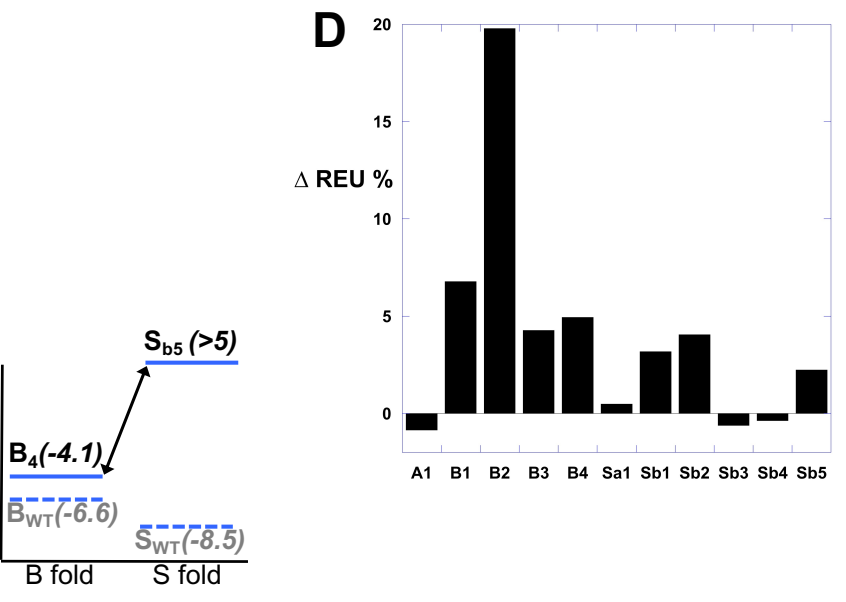

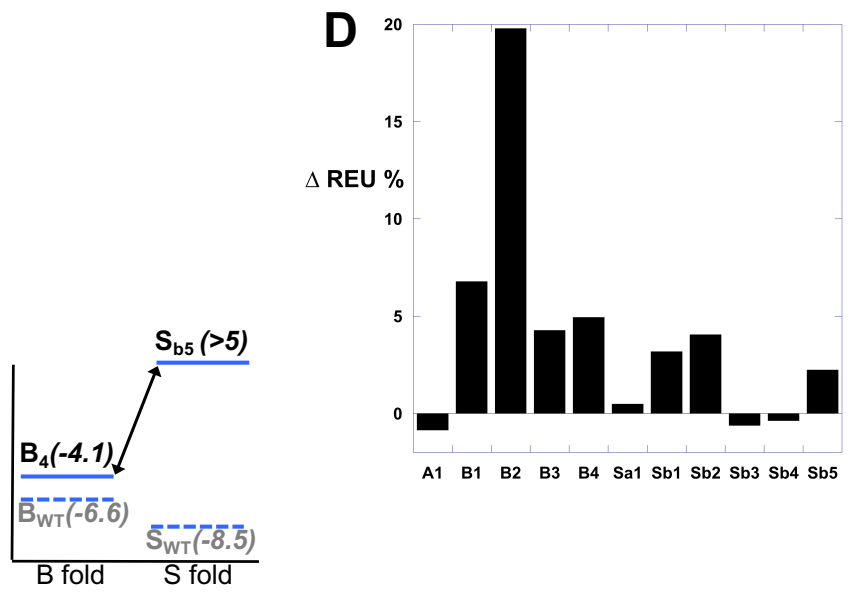

Figure 4: Energetics of fold switches. (A) $\Delta G$ vs $T$ profiles for 56 residue proteins. (B) $\Delta G$ vs $T$ profiles for longer proteins. $\Delta G_{\text {folding }}$ is plotted vs temperature in order to assess stability at a reference state of $298^{\circ} \mathrm{K}$. The curvature of the profiles reflects the $\Delta \mathrm{Cp}$ of folding for each protein (41). $\Delta \mathrm{Cp}=-0.69 \mathrm{kcal} /{ }^{\circ} \mathrm{mol}$ for $\mathrm{G}_{B}$ and $-0.26 \mathrm{kcal} /{ }^{\circ} \mathrm{mol}$ for $\mathrm{G}_{\mathrm{A}}$. $(42,44)$ and $-1.1 \mathrm{kcal} /{ }^{\circ} \mathrm{mol}$ for $\mathrm{S} 6$. (C) Each panel shows the $\Delta G_{\text {folding }}$ for the 56 residue $\mathrm{G}$-fold and longer $S$-fold in a dimorphic pair. For example, the 56 residue $A_{1}$ protein has a $\Delta G_{\text {folding }}$ of $-4.0 \mathrm{kcal} / \mathrm{mol}$ for the A-fold. The same 56 residues in the longer $S_{a 1}$ protein has a $\Delta G_{\text {folding }}$ of $-7.5 \mathrm{kcal} / \mathrm{mol}$ for the $S$-fold. The green connecting arrows indicate a complete fold switch for these sequence pairs. (D) The percent change in Rosetta Energy Units (REU) between the parent protein and the designed switch protein is plotted. The computational design of $A_{1}$ was compared to the relaxed structure of a highly stable A-fold (43). The designs of $\mathrm{B}_{1}, \mathrm{~B}_{2}, \mathrm{~B}_{3}$, and $\mathrm{B}_{4}$ were compared with a highly stable $\mathrm{B}$ fold (44). The designs of $S_{a 1}, S_{b 1}, S_{b 2}, S_{b 3}, S_{b 4}$, and $S_{b 5}$ were compared to a highly stable S-fold (32). All designed proteins have relatively small changes in REU except for $B_{2}$. The $20 \%$ increase in REU for $B_{2}$ is consistent with its low stability. 

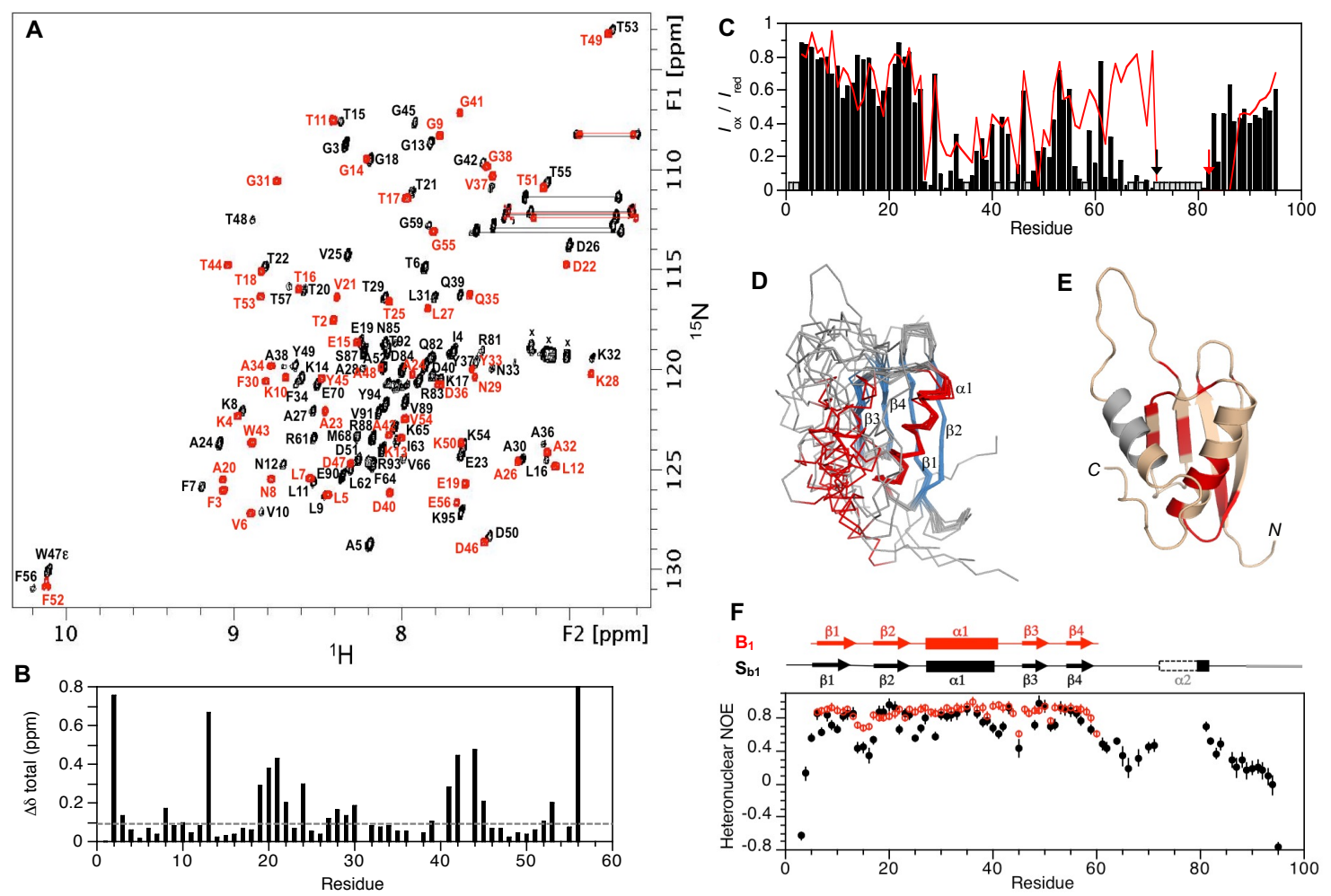

Figure 5: Comparison of $B_{1}$ and $S_{b 1}$. (A) Overlaid two dimensional ${ }^{1} \mathrm{H}_{-}{ }^{15} \mathrm{~N} H S Q C$ spectra of $S_{b 1}$ (black) and $\mathrm{B}_{1}$ (red) with backbone amide assignments. Spectra were recorded at $283^{\circ} \mathrm{K}$. (B) Plot of chemical shift perturbations between $\mathrm{S}_{\mathrm{b} 1}$ and $\mathrm{B}_{1}$ for backbone amides in the 56 amino acid identical region. Residue numbering is for $B_{1}$. (C) Plot of lox $/$ red versus residue for $S_{b 1-R 72 C-}$ MTSL (black) and $\mathrm{S}_{\mathrm{b} 1}-\mathrm{R} 83 \mathrm{C}-\mathrm{MTSL}$ (red). Gray columns indicate unassigned residues or prolines. The positions of the spin labels are indicated with arrows. (D) Ensemble of 10 lowest energy CSRosetta structures for $S_{b 1}$ using PRE restraints. (E) Cartoon representation of model 1 from the ensemble. Values of $\Delta \delta_{\text {total }}>0.1 \mathrm{ppm}$ from (B) are mapped onto the structure (red). Unassigned residues in the putative $\alpha 2$-helix are in gray. (F) Plot of $\left\{{ }^{1} \mathrm{H}\right\}-{ }^{15} \mathrm{~N}$ steady state heteronuclear NOE values at $600 \mathrm{MHz}$ versus residue for $\mathrm{B}_{1}$ (red) and for $\mathrm{S}_{\mathrm{b} 1}$ (black). Error bars indicate $\pm 1 \mathrm{SD}$. 


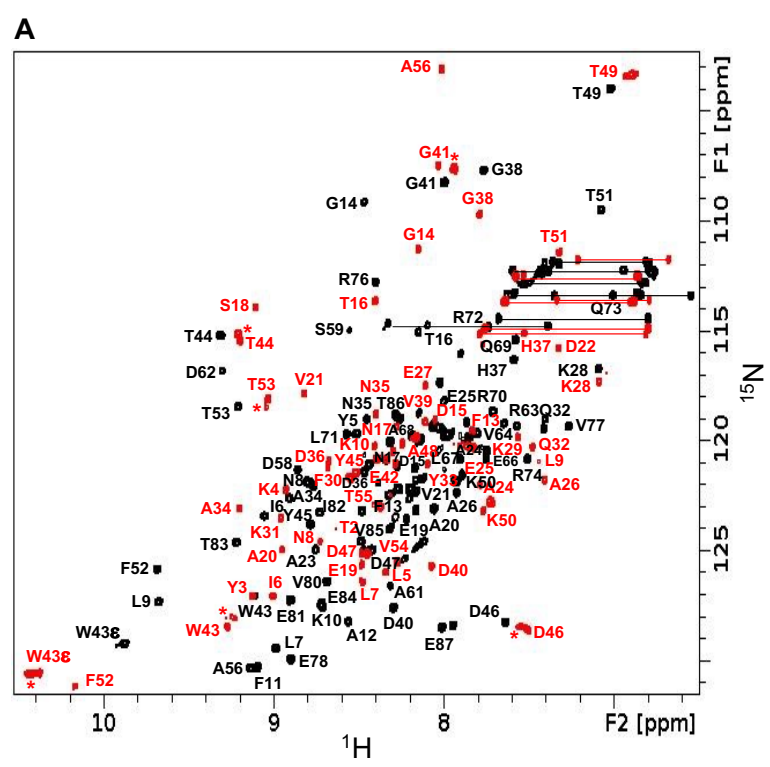

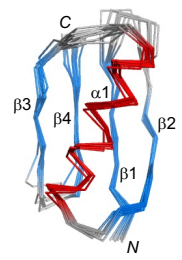

C
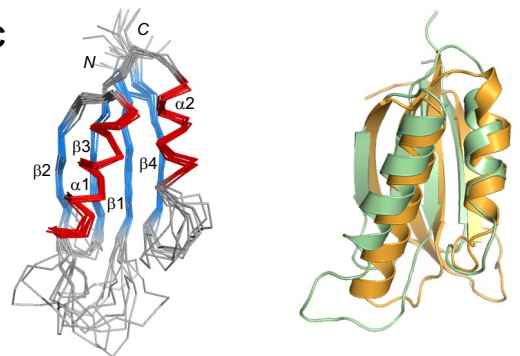

D

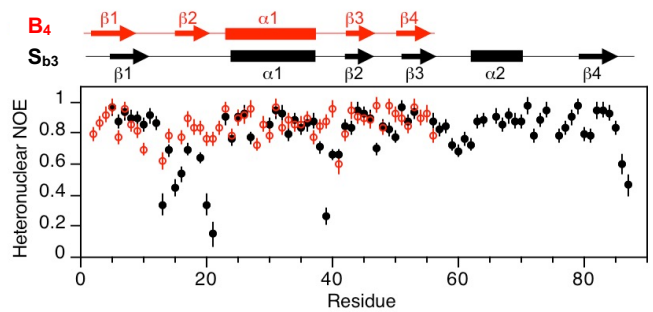

Figure 6: Structure and dynamics of $\mathrm{S}_{\mathrm{b} 3}$ and $\mathrm{B}_{4}$. (A) Overlaid two dimensional ${ }^{1} \mathrm{H}-{ }^{15} \mathrm{~N} \mathrm{HSQC}$ spectra of $\mathrm{S}_{\mathrm{b} 3}$ (black) and $\mathrm{B}_{4}(\mathrm{red})$ with backbone amide assignments. Spectra were recorded at $298^{\circ} \mathrm{K}$. The A56 peak is an aliased signal. Peaks labeled with an asterisk decrease in relative intensity as the $\mathrm{B}_{4}$ concentration is lowered, indicating the presence of a weakly associated putative dimer in addition to monomer. (B) Ensemble of 10 lowest energy CS-Rosetta structures for $\mathrm{B}_{4}$ (left panel). Superposition of the designed $\mathrm{B}_{4}$ structure (green) with the parent $\mathrm{G}_{\mathrm{B}}$ fold (orange) (right panel). (C) Ensemble of 10 lowest energy CS-Rosetta structures for $\mathrm{S}_{\mathrm{b} 3}$ (left panel). Superposition of $\mathrm{S}_{\mathrm{b} 3}$ (green) with the parent $\mathrm{S} 6$ fold (orange) (right panel). (D) Plot of $\left\{{ }^{1} \mathrm{H}\right\}-$ ${ }^{15} \mathrm{~N}$ steady state heteronuclear NOE values at $600 \mathrm{MHz}$ versus residue for $\mathrm{B}_{4}(\mathrm{red})$ and $\mathrm{S}_{\mathrm{b} 3}$ (black). Error bars indicate $\pm 1 \mathrm{SD}$. 
A
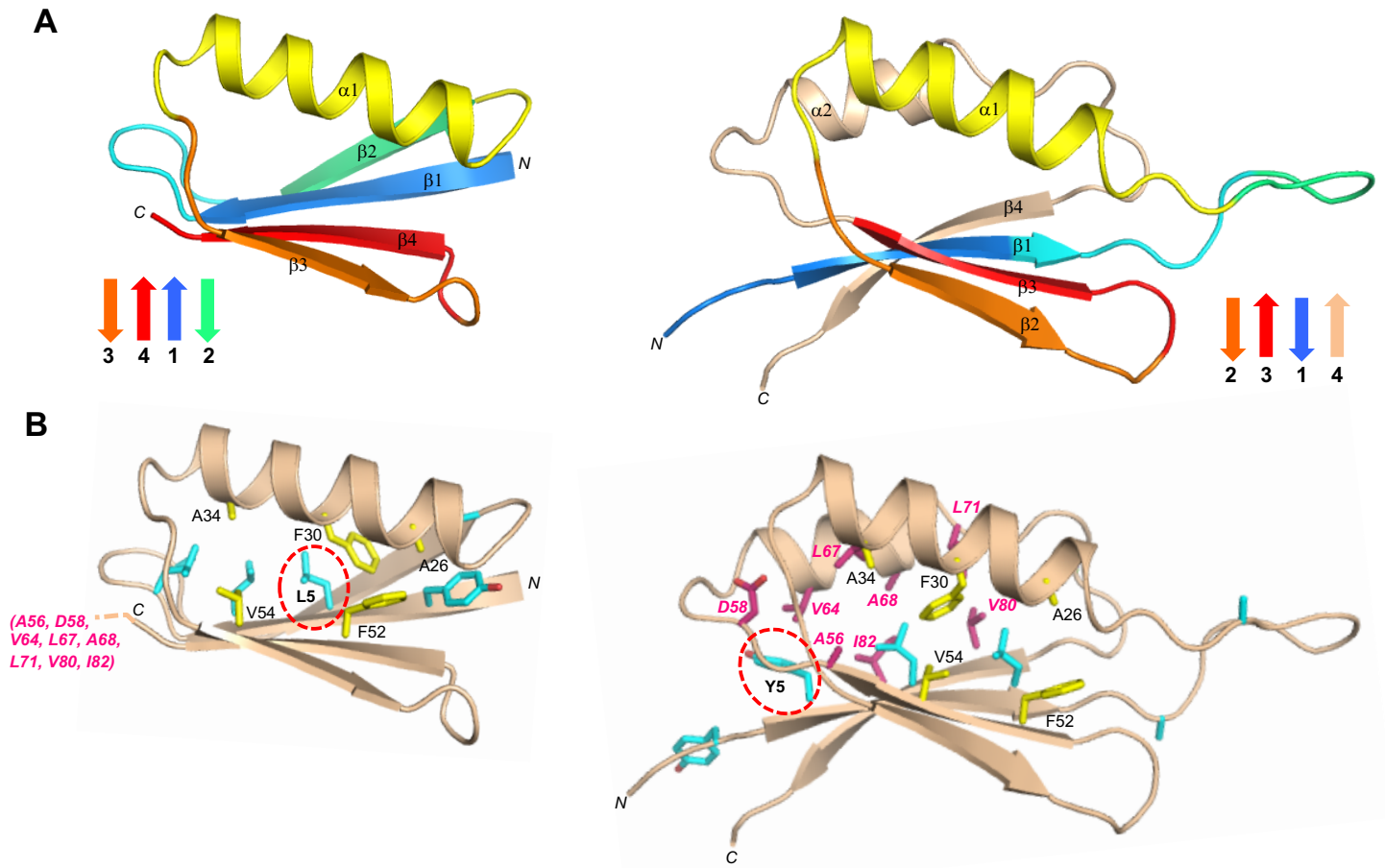

Figure 7: Structural differences in the high sequence identity regions of $B_{4}$ and $S_{b 3}$. (A) Main chain comparisons. (Left panel) CS-Rosetta structure of $\mathrm{B}_{4}$ with secondary structure elements color coded. (Right panel) Corresponding color-coded regions mapped onto the CS-Rosetta structure of $\mathrm{S}_{\mathrm{b} 3}$, showing changes in backbone conformation. Regions outside the 56 amino acid sequence of $B_{4}$ are shown in wheat. (B) Side chain comparisons. (Left panel) Residues contributing to the core of $B_{4}$ from $\alpha 1 / \beta 3 / \beta 4$ (yellow), and from other regions (cyan). The non$\alpha 1 / \beta 2 / \beta 3$ core residues from $S_{b 3}$ (pink) do not overlap with the $B_{4}$ core (see text for further details). (Right panel) Residues contributing to the core of $S_{b 3}$ from $\alpha 1 / \beta 2 / \beta 3$ (yellow), and the other participating core residues (pink). The non- $\alpha 1 / \beta 2 / \beta 3$ core residues from $B_{4}$ are also shown (cyan). The single L5Y amino acid difference between $\mathrm{B}_{4}$ and $\mathrm{S}_{\mathrm{b} 3}$ is highlighted. 

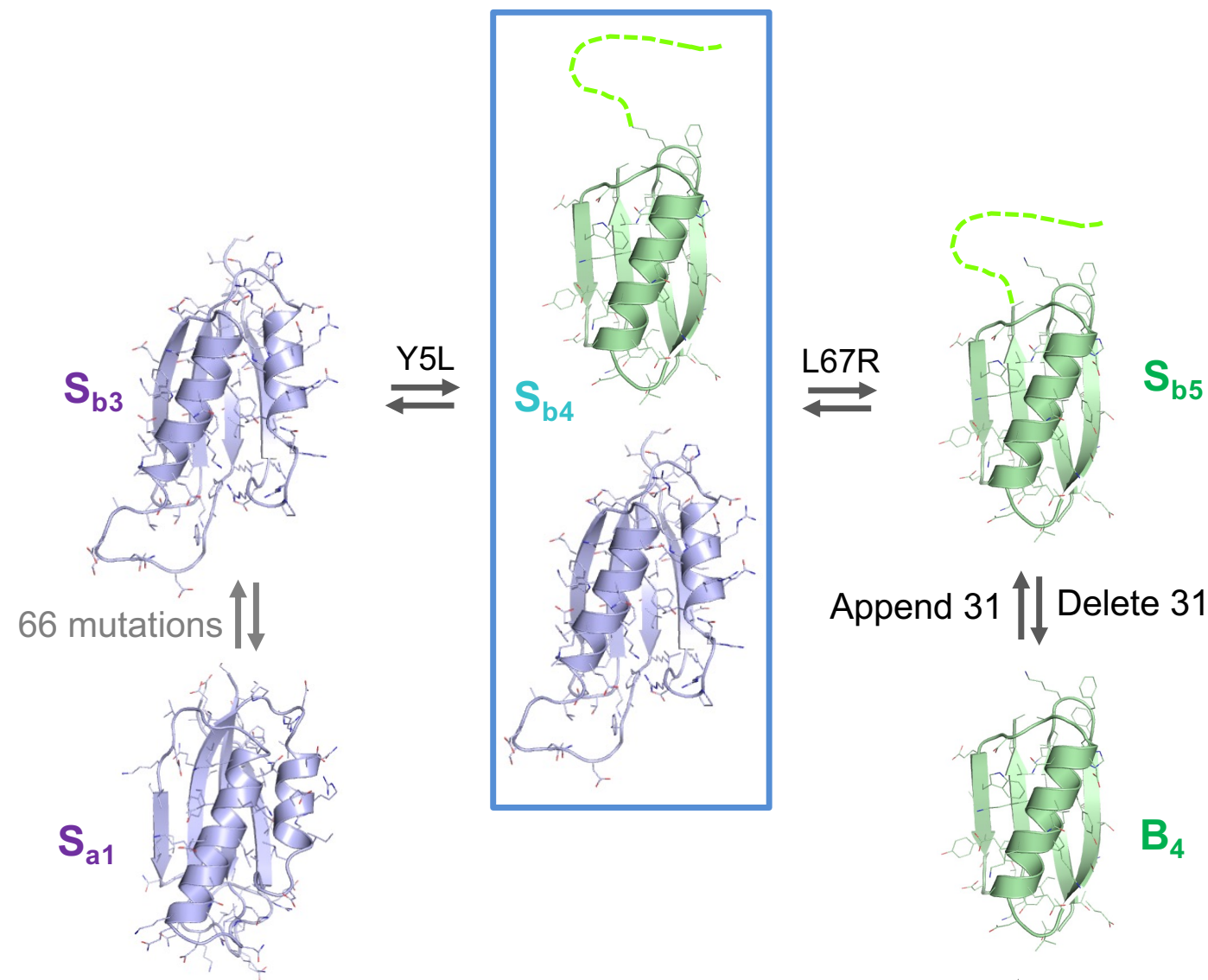

Append $\left.31\right|_{\downarrow}$ Delete 31
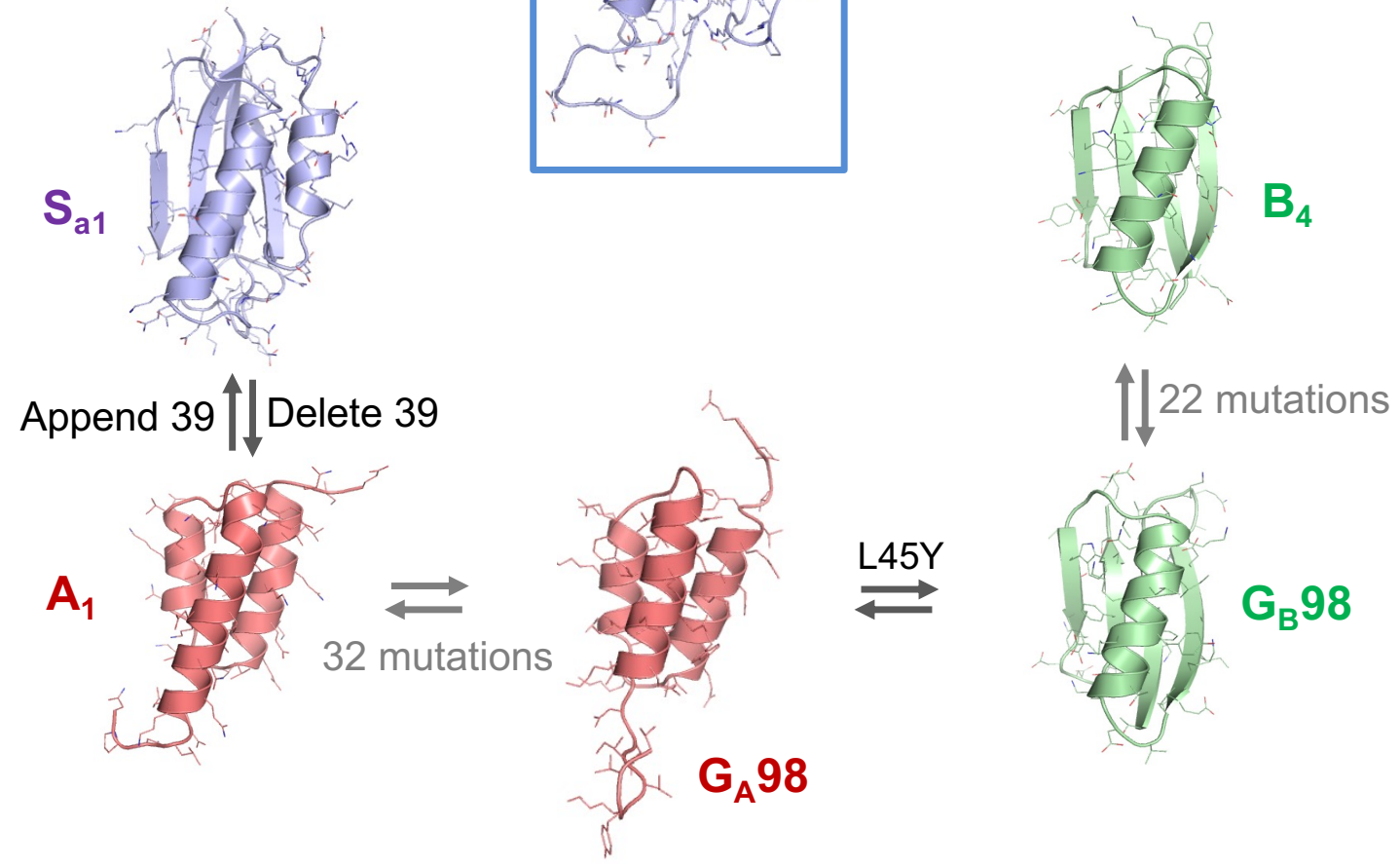

Figure 8: Sequence-fold relationships of engineered S-, A-, and B-folds. Switches between stable folds can be induced by point mutation or deleting/appending the part 2 sequence. Blue denotes an S-fold, green a B-fold, and red an A-fold. Gray arrows connect proteins that have been reengineered without a fold switch. $\mathrm{S}_{\mathrm{b} 4}$ (blue box) populates two folds simultaneously. The $\mathrm{G}_{\mathrm{A}} 98$ and $\mathrm{G}_{\mathrm{B}} 98$ proteins were described in an earlier paper (8). 